\title{
Global attractor for the Navier-Stokes equations with fractional deconvolution
}

\author{
Davide Catania, Alessandro Morando and Paola Trebeschi
}

\begin{abstract}
We consider a large eddy simulation model for the 3D NavierStokes equations obtained through fractional deconvolution of generic order. The global well-posedness of such a problem is already known. We prove the existence of the global attractor for the solution operator and find estimates for its Hausdorff and fractal dimensions both in terms of the Grashoff number and in terms of the mean dissipation length, with particular attention to the dependence on the fractional and deconvolution parameters. These results can be interpreted as bounds for the number of degrees of freedom of long-time dynamics, thus providing further information on the validity of the model for the simulation of turbulent $3 \mathrm{D}$ flows.
\end{abstract}

Mathematics Subject Classification. 76D05, 35Q30, 76F65, 76D03.

Keywords. Navier-Stokes equations, Global attractor, Fractal and Hausdorff dimension, Approximate deconvolution models (ADM) and methods, Fractional filter, Large eddy simulation (LES).

\section{Introduction}

We consider an approximate model for the Navier-Stokes Equations for a homogeneous incompressible fluid, that read

$$
\begin{aligned}
& \partial_{t} \boldsymbol{u}+\nabla \cdot(\boldsymbol{u} \otimes \boldsymbol{u})-\nu \Delta \boldsymbol{u}+\nabla \pi=\boldsymbol{f}, \\
& \nabla \cdot \boldsymbol{u}=0, \\
& \boldsymbol{u}(0, \cdot)=\boldsymbol{u}_{0},
\end{aligned}
$$

in $[0, T] \times D$, where $D$ is a three-space dimensional periodic domain under suitable conditions that we are going to make precise. We assume that the constant $\nu$, called kinematic viscosity, is positive. 
It is well-known that, currently, we lack a good well-posedness theory for these equations is lacking, so that this system is not suited for numerical simulations. Moreover, the simulation of turbulent pointwise flows is prohibitively expensive and sensitive to small perturbations of the data, i.e. the pointwise flow is chaotic. Large eddy simulation may overcome these difficulties (see the introductions in $[2,6]$ ). Instead of pointwise flows, averaged deterministic flows are considered, and systems satisfied by averages are studied. More precisely, some approximations are introduced in order to mitigate the nonlinearity and obtain well-posedness and the possibility to perform simulations. In particular, we are interested in fractional approximate deconvolution models (ADM), introduced by Stolz-Adams in [18].

We introduce the fractional filter, denoted by "- ", setting

$$
A_{\theta}=I+\alpha^{2 \theta}(-\Delta)^{\theta}, \quad \overline{\boldsymbol{v}}=A_{\theta}^{-1} \boldsymbol{v}
$$

(with periodic conditions in $\boldsymbol{x}$ ), where $\alpha>0$ and $\theta \in[0,1]$ are fixed. Let us note that the filter is linear and commutes with differentiation. It is a generalization of the Helmholtz filter, which corresponds to the choice $\theta=1$, and it is known to produce excellent results in numerical simulations, at least for large Reynolds numbers and suitable geometries (see [15] and the references therein).

Filtering the equations for $\boldsymbol{u}$, we obtain

$$
\begin{aligned}
& \partial_{t} \overline{\boldsymbol{u}}+\nabla \cdot \overline{(\boldsymbol{u} \otimes \boldsymbol{u})}-\nu \Delta \overline{\boldsymbol{u}}+\nabla \bar{\pi}=\overline{\boldsymbol{f}}, \\
& \nabla \cdot \overline{\boldsymbol{u}}=0, \\
& \overline{\boldsymbol{u}}(0, \cdot)=\overline{\boldsymbol{u}}_{0} .
\end{aligned}
$$

Set $\boldsymbol{w}=\overline{\boldsymbol{u}}$ and $q=\bar{\pi}$, so that $\boldsymbol{u}=A_{\theta} \boldsymbol{w}$. We solve the so-called interior closure problem by the approximation

$$
\overline{\boldsymbol{u} \otimes \boldsymbol{u}} \approx \overline{D_{N, \theta} \overline{\boldsymbol{u}} \otimes D_{N, \theta} \overline{\boldsymbol{u}}}=\overline{D_{N, \theta} \boldsymbol{w} \otimes D_{N, \theta} \boldsymbol{w}},
$$

where we have used the fractional deconvolution operator

$$
D_{N, \theta} \boldsymbol{w}:=\sum_{h=0}^{N}\left(I-A_{\theta}^{-1}\right)^{h} \boldsymbol{w},
$$

defined for every integer $N \geq 0$ and any real $\theta \in[0,1]$.

We finally deduce the fractional ADM

$$
\begin{aligned}
& \partial_{t} \boldsymbol{w}+\nabla \cdot \overline{\left(D_{N, \theta} \boldsymbol{w} \otimes D_{N, \theta} \boldsymbol{w}\right)}-\nu \Delta \boldsymbol{w}+\nabla q=\overline{\boldsymbol{f}}, \\
& \nabla \cdot \boldsymbol{w}=0 \\
& \boldsymbol{w}(0, \cdot)=\overline{\boldsymbol{u}}_{0} .
\end{aligned}
$$

This model has been introduced by Berselli-Lewandowski [4], where global well-posedness for $\theta>3 / 4$ and convergence results are proved (see Sect. 2.2 for the existence). This result has been generalized by Ali [1] to the case of magnetohydrodynamics requiring only $\theta \geq 1 / 2$.

When the deconvolution order is 0 , i.e. $N=0$, and the power $\theta$ is nonfractional, i.e. 1, we obtain the model introduced by Layton-Lewandowski [13] and studied by Cao-Lunasin-Titi [5], who prove the existence of global solutions 
and of the global attractor (see Sect. 4) for the solution operator, providing also estimates of its fractal and Hausdorff dimensions. These notions are useful in order to test the validity of the model as a large eddy simulation model. Actually, the dimension of the global attractor can be interpreted as the number of degrees of freedom of the long-time dynamics of the system.

In this paper, we are interested in extending such results to the fractional deconvolution model (3)-(5), with particular concern to the dependence on the fractional power $\theta$. First, we prove the existence of the global attractor associated to the semigroup generated by the operator $S(t)$ that associates to the initial datum $\mathbf{u}_{\mathbf{0}}$ the corresponding solution of $(3)-(4)$ evaluated at time $t$ (see Sect. 2.3 for the definition of the function space $\mathbf{H}_{\sigma}^{\theta}$ ).

Theorem 1.1. Assume that $\theta \geq 1 / 2$ and let $(S(t))_{t \geq 0}$ be the semigroup in $\mathbf{H}_{\sigma}^{\theta}$ associated to the initial value problem (3)-(5) defined in (28), with datum $\boldsymbol{f} \in \boldsymbol{H}^{-1}$. Then a unique global attractor $\mathcal{A}$ exists for such a semigroup in $\mathbf{H}_{\sigma}^{\theta}$.

The proof of Theorem 1.1 is given in Sect. 4. Note that the existence of solutions to (3)-(5) (see Sect. 2.2) is known just for $\theta \geq 1 / 2$, whose assumption in our result, for this reason, is not restrictive.

The subsequent step consists in finding estimates for the attractor $\mathcal{A}$. We resort to the trace formula and the standard technique described for instance in $[10,19]$. This approach requires that the operator $S(t)$ is Fréchet differentiable with respect to the initial datum. After proving this result (see Sect. 5), we introduce a suitable version of the Grashoff number adapted to the model we are considering:

$$
G:=\frac{L^{3 / 2}\left\|A_{\theta}^{-1 / 2} D_{N, \theta}^{1 / 2} \boldsymbol{f}\right\|}{\nu^{2}} .
$$

First, we prove an estimate for the global attractor dimension in terms of this number.

Theorem 1.2. If $\boldsymbol{f} \in \boldsymbol{H}^{-\theta}$ for $\theta \in(1 / 2,1]$, the Hausdorff dimension $d_{\mathrm{H}}(\mathcal{A})$ and the fractal dimension $d_{\mathrm{F}}(\mathcal{A})$ of the global attractor $\mathcal{A}$ are bounded above by

$$
d_{\mathrm{H}}(\mathcal{A}) \leq d_{\mathrm{F}}(\mathcal{A}) \leq C\left(C_{1} \nu^{1+2 \mu} L^{1+\mu}\right)^{\frac{3}{5+3(\sigma-1) s}} G^{\frac{6+6 \mu}{5+3(\sigma-1) s}},
$$

where $C$ is a numerical constant independent of the other parameters appearing in the right-hand side, $C_{1}$ is defined in (72), $\sigma$ is defined in (94), while $\mu$ and $s$ are defined in (73).

The Grashoff number takes into account the effect of the forcing term $\boldsymbol{f}$, so that the previous theorem establishes a relation between the global attractor dimension and $\boldsymbol{f}$.

Notice that, in view of the definition (94) of $\sigma$, it is convenient to analyze the estimate (7) by considering separately two cases corresponding to the different ranges $(1 / 2,2 / 3)$ and $[2 / 3,1]$ for $\theta$.

(i) For $2 / 3 \leq \theta \leq 1$ we have $(\sigma-1) s=0$; then the bound in the right-hand side reduces to

$$
C\left(C_{1} \nu^{1+2 \mu} L^{1+\mu}\right)^{\frac{3}{5}} G^{\frac{6+6 \mu}{5}},
$$


where $\mu=\frac{2(1-\theta)}{2 \theta-1}[$ cf. (77)]. In particular, for $\theta=1$ we have $\mu=0$, hence the exponent of $G$ in (8) reduces to $6 / 5$ and we recover the already known estimate proved in [5] (but now it holds for all $N$ and not only for $N=0)$. We also observe that, when $\theta<1$, the exponent worsens.

(ii) For $1 / 2<\theta<2 / 3$ the result is worse with respect to the case (i), not only because of the values of $\theta$, but also since the exponent $(\sigma-1) s \neq 0$.

Now, in order to understand more deeply the relation between the global attractor dimension and the number of degrees of freedom of the long-time dynamics, we introduce the mean rate of energy (of the model) dissipation, defined by

$$
\bar{\varepsilon}:=\frac{1}{L^{3}} \sup _{\boldsymbol{w}_{0} \in \mathcal{A}} \limsup _{T \rightarrow+\infty} \frac{1}{T} \int_{0}^{T} \nu\left\|\nabla A_{\theta}^{1 / 2} D_{N, \theta}^{1 / 2} \boldsymbol{w}(t)\right\|^{2} d t .
$$

Moreover, in analogy with Kolmogorov dissipation length in the classical theory of turbulence, we define the mean dissipation length for the model as

$$
\ell_{d}:=\left(\frac{\nu^{3}}{\bar{\varepsilon}}\right)^{1 / 4}
$$

so that

$$
\sup _{\boldsymbol{w}_{0} \in \mathcal{A}} \limsup _{T \rightarrow+\infty} \frac{1}{T} \int_{0}^{T} \nu\left\|\nabla A_{\theta}^{1 / 2} D_{N, \theta}^{1 / 2} \boldsymbol{w}(t)\right\|^{2} d t=\frac{L^{3} \nu^{3}}{\ell_{d}^{4}} .
$$

Theorem 1.3. If $\boldsymbol{f} \in \boldsymbol{H}^{-1}$ and $\theta \in(1 / 2,1]$, the Hausdorff dimension $d_{\mathrm{H}}(\mathcal{A})$ and the fractal dimension $d_{\mathrm{F}}(\mathcal{A})$ of the global attractor $\mathcal{A}$ are bounded above by

$$
d_{\mathrm{H}}(\mathcal{A}) \leq d_{\mathrm{F}}(\mathcal{A}) \leq C\left[(N+1)^{\gamma}\left(\frac{L}{\alpha}\right)^{2 \gamma \theta}\left(\frac{L}{\ell_{d}}\right)^{2}\right]^{\frac{6}{6 \gamma-1}}
$$

where $C$ is a numerical constant independent of the other parameters appearing in the right-hand side, and $\gamma$ is defined in (86).

Considerations similar to those for Theorem 1.2 still hold. In particular, when $\theta=1$, we have again that $\gamma=1$ and the exponent of $\left(L / \ell_{d}\right)$ reduces to $12 / 5$, exactly as in [5].

The mean dissipation length $\ell_{d}$ is defined to be the smallest length scale actively participating in the dynamics of the turbulent flow. This means that, when performing simulations, the size of every mesh can not be greater than $\ell_{d}$ and $L / \ell_{d}$ points (for each space dimension) are needed and the number of degrees of freedom for a $3 \mathrm{D}$ turbulent flow is expected to be $\left(L / \ell_{d}\right)^{3}$. The previous theorem shows the dependence of the number of degrees of freedom of the fractional deconvolution model with respect to $\theta$ and the other parameters. The effect of filtering is the reduction of this number (for $\theta=1$, we have $12 / 5$, which is less than 3$)$, and a reduced regularization $(\theta<1)$ increases the number of degrees of freedom. In particular, recalling the definition of $\gamma(86)$, we can easily compute that $\frac{12}{6 \gamma-1}=3$ holds for $\theta=9 / 10$. 
Despite the use of standard techniques, the proofs are not trivial, since the presence of the deconvolution operator of generic order $N$ and, above all, of the fractional power $\theta$ require a clever combination of several ad hoc estimates. When $\theta$ decreases, the regularization provided by the filter lessens, so that refined interpolation estimates are needed. Note that we do not compute the bound of the attractor dimension when $\theta=1 / 2$; this is due to the fact that, in such a case, $1+\theta=3 / 2$, and it is impossible to apply the fractional Agmon's inequality (Lemma A.3) in order to estimate appropriately some $L^{\infty}$-norms by using (104).

We are convinced that the theorems proved in this paper can be extended to other models, even for magnetofluids, generalizing the results concerning the global attractor in magnetohydrodynamics (MHD), as in [7-9], to deconvolution and fractional deconvolution approximate MHD models, such as those in $[1,3]$. However, this would require several more computations, and it is postponed to future works.

Outline of the paper Section 2 is devoted to present the main notation, function spaces, the notion of regular weak solution, the properties of the filter and of the deconvolution operator. In Sect. 3 we prove some a priori estimates that will be used in the proofs of the main theorems. In Sect. 4 we recall some notions and properties concerning global attractors and prove Theorem 1.1. Section 5 is devoted to the proof of the differentiability of the solution operator, while Sect. 6 contains all results concerning the bounds of the global attractor dimension, i.e. Theorems 1.2 and 1.3. Finally, the Appendix lists and proves some useful formulas and inequalities that play a crucial role in the proofs of the original results contained in this paper.

\section{Preliminary results and basic tools}

\subsection{Notation}

We set

$$
\begin{aligned}
& \boldsymbol{x}=\left(x_{1}, x_{2}, x_{3}\right), \\
& \partial_{j}=\partial_{x_{j}}, \quad \Delta=\partial_{1}^{2}+\partial_{2}^{2}+\partial_{3}^{2}, \quad \nabla=\left(\partial_{1}, \partial_{2}, \partial_{3}\right) .
\end{aligned}
$$

The space domain is $\mathbb{T}_{3}=\left\{\boldsymbol{x} \in \mathbb{R}^{3}:-\pi L<x_{1}, x_{2}, x_{3}<\pi L\right\}, L>0$, with $2 \pi L$ periodicity with respect to $\boldsymbol{x}$ (i.e. with respect to $x_{1}, x_{2}, x_{3}$ ), i.e. a torus.

We denote by $\mathrm{L}^{p}$ and $\mathrm{H}^{s}$ (with $1 \leq p \leq \infty$ and $s \in \mathbb{R}$ ) the standard Lebesgue and Sobolev spaces, and define $\mathscr{C}([0, T] ; X)$ and the Bochner spaces $\mathrm{L}^{p}(0, T ; X)$ in the usual way. We write $\mathbf{H}^{s}$ for the Sobolev space of vector functions defined on $\mathbb{T}_{3}$ with zero spatial mean and $2 \pi L$ periodicity in $\boldsymbol{x}$, and set $\mathbf{L}^{2}=\mathbf{H}^{0}$. We denote by the subscript $\sigma$ spaces of divergence-free (classes of) functions, and we assume the domain $\mathbb{T}_{3}$ when not specified. The symbol $(\cdot, \cdot)$ is used to denote the standard scalar product in $\mathbf{L}^{2}$.

We will denote by $C$ a generic positive constant, independent of the relevant parameters, which may differ at every occurrence, even in the same expression. 


\subsection{Regular weak solutions}

Assume that $\boldsymbol{f}=\boldsymbol{f}(\boldsymbol{x}) \in \mathbf{H}^{-3 / 2}$ is independent of time (for simplicity), so that $\overline{\boldsymbol{f}} \in \mathbf{H}^{2 \theta-3 / 2}$, and $\boldsymbol{u}_{0} \in \mathbf{H}_{\sigma}^{-\theta}$, so that $\boldsymbol{w}_{0}:=\overline{\boldsymbol{u}}_{0} \in \mathbf{H}_{\sigma}^{\theta}$ (we may even require the stronger regularity $\boldsymbol{u}_{0} \in \mathbf{L}_{\sigma}^{2}$, which is more natural for the unfiltered problem).

We say that $\boldsymbol{w}:[0,+\infty) \times \mathbb{T}^{3} \rightarrow \mathbb{R}^{3}$ is a regular weak solution of (3)-(5) when, for all $T>0$, the following properties are verified (see also [12]).

- Regularity:

$$
\begin{aligned}
& \boldsymbol{w} \in \mathscr{C}\left([0, T] ; \mathbf{H}_{\sigma}^{\theta}\right) \cap \mathrm{L}^{2}\left(0, T ; \mathbf{H}_{\sigma}^{1+\theta}\right), \\
& \partial_{t} \boldsymbol{w} \in \mathrm{L}^{2}\left(0, T ; \mathbf{H}_{\sigma}^{2 \theta-3 / 2}\right) \\
& q \in \mathrm{L}^{2}\left(0, T ; \mathrm{H}^{2 \theta-1 / 2}\right) .
\end{aligned}
$$

- Initial data: $\boldsymbol{w}(0, \cdot)=\boldsymbol{w}_{0}$.

- Weak formulation:

$$
\begin{aligned}
& \int_{0}^{+\infty}\left\{\left(\boldsymbol{w}, \partial_{t} \boldsymbol{\varphi}\right)-\nu(\nabla \boldsymbol{w}, \nabla \boldsymbol{\varphi})+\left(D_{N, \theta} \boldsymbol{w} \otimes D_{N, \theta} \boldsymbol{w}, \nabla \overline{\boldsymbol{\varphi}}\right)+q \nabla \cdot \boldsymbol{\varphi}\right\}(s) \mathrm{d} s \\
& =-\int_{0}^{+\infty}(\boldsymbol{f}, \overline{\boldsymbol{\varphi}})(s) \mathrm{d} s-(\boldsymbol{w}(0), \boldsymbol{\varphi}(0)) \\
& \quad \text { for each } \boldsymbol{\varphi} \in\left(\mathscr{C}_{0}^{\infty}\left(\mathbb{T}^{3} \times[0, T)\right)\right)^{3} .
\end{aligned}
$$

Theorem 2.1. Assume $\theta \geq 1 / 2$. Then there exists a unique regular weak solution of (3)-(5). This solution depends continuously on the data (the system is well-posed) and satisfies the energy (of the model) identity

$$
\begin{aligned}
& \frac{1}{2}\left\|A_{\theta}^{1 / 2} D_{N, \theta}^{1 / 2} \boldsymbol{w}(t)\right\|^{2}+\nu \int_{0}^{t}\left\|\nabla A_{\theta}^{1 / 2} D_{N, \theta}^{1 / 2} \boldsymbol{w}(s)\right\|^{2} \mathrm{~d} s \\
& =\frac{1}{2}\left\|A_{\theta}^{1 / 2} D_{N, \theta}^{1 / 2} \boldsymbol{w}(0)\right\|^{2}+\int_{0}^{t}\left(D_{N, \theta}^{1 / 2} \boldsymbol{f}, D_{N, \theta}^{1 / 2} \boldsymbol{w}(s)\right) \mathrm{d} s
\end{aligned}
$$

for each $t \in[0,+\infty)$.

This result has been proved by Berselli-Lewandowski [4] for $\theta>3 / 4$ and extended to $\theta \geq 1 / 2$ by Ali in [1], where the equations are coupled with the equations for the magnetic field (so the theorem above follows taking the magnetic field identically zero).

\subsection{More on functional spaces}

Let $L>0$ be given. Let $\mathbb{T}^{3}$ be the torus defined as the compact quotient manifold, $\mathbb{T}^{3}=\mathbb{R}^{3} / 2 \pi L \mathbb{Z}^{3}$.

For general $s \in \mathbb{R}$, we write $\mathbf{H}^{s}$ for the Sobolev space of vector functions defined on $\mathbb{T}^{3}$ with zero spatial mean, i.e.

$$
\mathbf{H}^{s}:=\left\{\boldsymbol{w}: \mathbb{T}^{3} \rightarrow \mathbb{R}^{3}, \boldsymbol{w} \in\left[\mathrm{H}^{s}\left(\mathbb{T}^{3}\right)\right]^{3}, \int_{\mathbb{T}^{3}} \boldsymbol{w} \mathrm{d} \boldsymbol{x}=0\right\},
$$

and set $\mathbf{L}^{2}=\mathbf{H}^{0}$. 
We denote by the subscript $\sigma$ spaces of divergence-free (classes of) functions, i.e.

$$
\mathbf{H}_{\sigma}^{s}:=\left\{\boldsymbol{w}: \mathbb{T}^{3} \rightarrow \mathbb{R}^{3}, \boldsymbol{w} \in\left[\mathrm{H}^{s}\left(\mathbb{T}^{3}\right)\right]^{3}, \nabla \cdot \boldsymbol{w}=0, \quad \int_{\mathbb{T}^{3}} \boldsymbol{w} \mathrm{d} \boldsymbol{x}=0\right\} .
$$

Since we work with periodic boundary conditions we can describe the spaces that we use in terms of the Fourier series on the $3 D$ torus $\mathbb{T}^{3}$. A vector field $\boldsymbol{w} \in \mathbf{H}_{\sigma}^{s}$ can be expanded in terms of Fourier series (of $2 \pi L$-periodic functions) as

$$
\boldsymbol{w}(\boldsymbol{x})=\sum_{\boldsymbol{k} \in \mathcal{T}_{3}^{*}} \widehat{\boldsymbol{w}}_{\boldsymbol{k}} \mathrm{e}^{\mathrm{i} \boldsymbol{k} \cdot \boldsymbol{x}},
$$

where the space of frequencies $\mathcal{T}_{3}^{*}$ is defined by setting

$$
\mathcal{T}_{3}:=\frac{2 \pi}{2 \pi L} \mathbb{Z}^{3}=\frac{1}{L} \mathbb{Z}^{3} \quad \text { and } \quad \mathcal{T}_{3}^{*}:=\mathcal{T}_{3} \backslash\{\mathbf{0}\}
$$

and the Fourier coefficients are given $b^{1}$

$$
\widehat{\boldsymbol{w}}_{\boldsymbol{k}}=\frac{1}{(2 \pi L)^{3}} \int_{\mathbb{T}_{3}} \boldsymbol{w}(\boldsymbol{x}) \mathrm{e}^{-\mathrm{i} \boldsymbol{k} \cdot \boldsymbol{x}} d \boldsymbol{x} .
$$

We set $|\boldsymbol{k}|:=\sqrt{\left|k_{1}\right|^{2}+\left|k_{2}\right|^{2}+\left|k_{3}\right|^{2}}$, and we introduce the $\mathbf{H}^{s}$ norm by

$$
\|\boldsymbol{w}\|_{s, 2}^{2}=\sum_{\boldsymbol{k} \in \mathcal{T}_{3}^{*}}|\boldsymbol{k}|^{2 s}\left|\widehat{\boldsymbol{w}}_{\boldsymbol{k}}\right|^{2}
$$

(for $s=0$ we set $\|\cdot\|:=\|\cdot\|_{0,2}$ ). The inner product associated with this norm is

$$
(\boldsymbol{w}, \boldsymbol{v})_{\mathbf{H}^{s}}=\sum_{\boldsymbol{k} \in \mathcal{T}_{3}^{*}}|\boldsymbol{k}|^{2 s}\left(\widehat{\boldsymbol{w}}_{\boldsymbol{k}}, \widehat{\widehat{\boldsymbol{v}}}_{\boldsymbol{k}}\right)
$$

where here $\overline{\widehat{\boldsymbol{v}}_{\boldsymbol{k}}}$ denotes the complex conjugate of $\widehat{\boldsymbol{v}}_{\boldsymbol{k}}$ (if $\widehat{\boldsymbol{v}}_{\boldsymbol{k}}=\left(v_{\boldsymbol{k}}^{1}, v_{\boldsymbol{k}}^{2}, v_{\boldsymbol{k}}^{3}\right)$ we have $\left.\overline{\boldsymbol{v}}_{\boldsymbol{k}}=\left(\overline{v_{\boldsymbol{k}}^{1}}, \overline{v_{\boldsymbol{k}}^{2}}, \overline{v_{\boldsymbol{k}}^{3}}\right)\right)$ and $(\cdot, \cdot)$ in the right-hand side of the above formula is the scalar product in $\mathbb{C}^{3}$.

Since we are looking for real vector valued functions, we have that the Fourier coefficients satisfy

$$
\widehat{\boldsymbol{v}}_{\boldsymbol{k}}=\overline{\widehat{\boldsymbol{v}}_{-\boldsymbol{k}}}, \quad \forall \boldsymbol{k} \in \mathcal{T}_{3}^{*} .
$$

Therefore, the spaces $\mathbf{H}^{s}$ can be rewritten as

$\mathbf{H}^{s}:=\left\{\boldsymbol{w}(\boldsymbol{x})=\sum_{\boldsymbol{k} \in \mathcal{T}_{3}^{*}} \widehat{\boldsymbol{w}}_{\boldsymbol{k}} \mathrm{e}^{\mathrm{i} \boldsymbol{k} \cdot \boldsymbol{x}}, \widehat{\boldsymbol{w}}_{\boldsymbol{k}}=\overline{\widehat{\boldsymbol{w}}_{-\boldsymbol{k}}},\|\boldsymbol{w}\|_{s, 2}^{2}=\sum_{\boldsymbol{k} \in \mathcal{T}_{3}^{*}}|\boldsymbol{k}|^{2 s}\left|\widehat{\boldsymbol{w}}_{\boldsymbol{k}}\right|^{2}<+\infty\right\}$.

\footnotetext{
${ }^{1}$ We note that $\widehat{\boldsymbol{w}}_{\mathbf{0}}=\frac{1}{(2 \pi L)^{3}} \int_{\mathbb{T}_{3}} \boldsymbol{w}(\boldsymbol{x}) d \boldsymbol{x}=0$ since $\boldsymbol{w}$ has zero mean.
} 


\subsection{Filter properties}

In this paper we will use the deconvolution operator $D_{N, \theta}$, defined by $(2)$ and studied in Sect. 2.5, which is constructed by using $A_{\theta}$ defined in (1), i.e. the Helmholtz operator with fractional regularization.

For real $p$ the pseudo-differential operator $A_{\theta}^{p}$ is defined in the periodic case as

$$
A_{\theta}^{p} \boldsymbol{w}:=\sum_{\boldsymbol{k} \in \mathcal{T}_{3}^{*}}\left(1+\alpha^{2 \theta}|\boldsymbol{k}|^{2 \theta}\right)^{p} \widehat{\boldsymbol{w}}_{\boldsymbol{k}} \mathrm{e}^{\mathrm{i} \boldsymbol{k} \cdot \boldsymbol{x}} .
$$

Lemma 2.2. (i) For all $p \geq 0$ the operator $A_{\theta}^{p}$, as a linear operator in $\mathbf{L}^{2}$, is self-adjoint and commutes with differentiation.

(ii) The inverse operator $A_{\theta}^{-1}$ is continuous from $\mathbf{H}^{s}$ to $\mathbf{H}^{s+2 \theta}$ with the estimate

$$
\left\|A_{\theta}^{-1} \boldsymbol{w}\right\|_{s+2 \theta, 2} \leq \alpha^{-2 \theta}\|\boldsymbol{w}\|_{s, 2}
$$

(iii) We have

$$
\left\|A_{\theta}^{1 / 2} \boldsymbol{w}\right\|^{2}=\|\boldsymbol{w}\|^{2}+\alpha^{2 \theta}\left\|(-\Delta)^{\theta / 2} \boldsymbol{w}\right\|^{2}, \quad \forall \boldsymbol{w} \in \mathbf{H}^{\theta} .
$$

Proof. For the proof of (i)-(ii) we refer to [1]. Concerning (iii), using that $A_{\theta}^{1 / 2}$ and $(-\Delta)^{\theta / 2}$ are self-adjoint, we get

$$
\begin{aligned}
\left\|A_{\theta}^{1 / 2} \boldsymbol{w}\right\|^{2} & =\left(A_{\theta}^{1 / 2} \boldsymbol{w}, A_{\theta}^{1 / 2} \boldsymbol{w}\right)=\left(\boldsymbol{w}, A_{\theta} \boldsymbol{w}\right) \\
& =\left(\boldsymbol{w},\left(I+\alpha^{2 \theta}(-\Delta)^{\theta}\right) \boldsymbol{w}\right)=\|\boldsymbol{w}\|^{2}+\alpha^{2 \theta}\left((-\Delta)^{\theta / 2} \boldsymbol{w},(-\Delta)^{\theta / 2} \boldsymbol{w}\right) \\
& =\|\boldsymbol{w}\|^{2}+\alpha^{2 \theta}\left\|(-\Delta)^{\theta / 2} \boldsymbol{w}\right\|^{2} .
\end{aligned}
$$

Remark 2.3. Since the relevant cases and applications concern small values of $\alpha$, from now on, we assume $0<\alpha \leq 1$; this makes some terms less cumbersome and does not limit the generality of the results.

Remark 2.4. Formula (11) implies the following equivalence of norms

$$
\frac{1}{\sqrt{2}}\left\|A_{\theta}^{1 / 2} \boldsymbol{w}\right\| \leq\|\boldsymbol{w}\|_{\theta, 2} \leq \frac{1}{\alpha^{\theta}}\left\|A_{\theta}^{1 / 2} \boldsymbol{w}\right\|, \quad \forall \boldsymbol{w} \in \mathbf{H}^{\theta} .
$$

By the same reason we have also that

$$
\frac{1}{2}\left\|A_{\theta} \boldsymbol{w}\right\| \leq\|\boldsymbol{w}\|_{2 \theta, 2} \leq \frac{1}{\alpha^{2 \theta}}\left\|A_{\theta} \boldsymbol{w}\right\|, \quad \forall \boldsymbol{w} \in \mathbf{H}^{2 \theta} .
$$

\subsection{The deconvolution operator}

We can express the pseudo-differential deconvolution operator in terms of the Fourier series. We have:

$$
D_{N, \theta} \boldsymbol{w}=\sum_{\boldsymbol{k} \in \mathcal{T}_{3}^{*}} \widehat{D}_{N, \theta}(\boldsymbol{k}) \widehat{\boldsymbol{w}}_{k} \mathrm{e}^{\mathrm{i} \boldsymbol{k} \cdot \boldsymbol{x}}
$$

where

$$
\widehat{D}_{N, \theta}(\boldsymbol{k})=\left(1+\alpha^{2 \theta}|\boldsymbol{k}|^{2 \theta}\right) \rho_{N, \boldsymbol{k}}, \quad \rho_{N, \boldsymbol{k}}=1-\left(\frac{\alpha^{2 \theta}|\boldsymbol{k}|^{2 \theta}}{1+\alpha^{2 \theta}|\boldsymbol{k}|^{2 \theta}}\right)^{N+1} .
$$


The following lemma is proved in [1].

Lemma 2.5. The symbol $\widehat{D}_{N, \theta}(\boldsymbol{k})$ of the operator $D_{N, \theta}$ satisfies the following properties:

$$
\begin{aligned}
& \widehat{D}_{0, \theta}(\boldsymbol{k})=1 \\
& 1 \leq \widehat{D}_{N, \theta}(\boldsymbol{k}) \leq N+1 \\
& \widehat{D}_{N, \theta}(\boldsymbol{k}) \leq \widehat{A}_{\theta}(\boldsymbol{k}):=\left(1+\alpha^{2 \theta}|\boldsymbol{k}|^{2 \theta}\right) .
\end{aligned}
$$

The following lemma is given in [4] (and [1]).

Lemma 2.6. For all $s \geq-1$ and $N>0$, the operator $D_{N, \theta}: \mathbf{H}^{s} \rightarrow \mathbf{H}^{s}$

- is self-adjoint;

- commutes with differentiation.

Moreover, for all $\boldsymbol{w}$ sufficiently smooth we have

$$
\|\boldsymbol{w}\|_{s, 2} \leq\left\|D_{N, \theta} \boldsymbol{w}\right\|_{s, 2} \leq\left\|A_{\theta}^{1 / 2} D_{N, \theta}^{1 / 2} \boldsymbol{w}\right\|_{s, 2} .
$$

Remark 2.7. In [1] the above inequalities are formulated in terms of a suitable constant $C$. In fact, one can take $C=1$, so we do not write $C$ in the above inequalities.

Lemma 2.8. For all $p \geq 0, s \geq-1$ and $N>0$, and for all $\boldsymbol{w}$ sufficiently smooth we have

$$
\|\boldsymbol{w}\|_{s, 2} \leq\left\|D_{N, \theta}^{p} \boldsymbol{w}\right\|_{s, 2} \leq(N+1)^{p}\|\boldsymbol{w}\|_{s, 2} .
$$

Proof. We use the definition of the norm (10) and (14) to have

$$
\begin{aligned}
\|\boldsymbol{w}\|_{s, 2}^{2} & =\sum_{\boldsymbol{k} \in \mathcal{T}_{3}^{*}}|\boldsymbol{k}|^{2 s}\left|\widehat{\boldsymbol{w}}_{\boldsymbol{k}}\right|^{2} \leq \sum_{\boldsymbol{k} \in \mathcal{T}_{3}^{*}}|\boldsymbol{k}|^{2 s}\left|\widehat{D}_{N, \theta}^{p}(\boldsymbol{k})\right|^{2}\left|\widehat{\boldsymbol{w}}_{\boldsymbol{k}}\right|^{2} \\
& \leq \sum_{\boldsymbol{k} \in \mathcal{T}_{3}^{*}}(N+1)^{2 p}|\boldsymbol{k}|^{2 s}\left|\widehat{\boldsymbol{w}}_{\boldsymbol{k}}\right|^{2}=(N+1)^{2 p}\|\boldsymbol{w}\|_{s, 2}^{2} .
\end{aligned}
$$

In the sequel we will make use of the following estimates which come from the estimates (15) and (16) as particular cases:

$$
\begin{aligned}
& \left\|D_{N, \theta} \boldsymbol{w}\right\| \leq\left\|A_{\theta}^{1 / 2} D_{N, \theta}^{1 / 2} \boldsymbol{w}\right\| ; \\
& \left\|A_{\theta}^{1 / 2} D_{N, \theta} \boldsymbol{w}\right\| \leq(N+1)^{1 / 2}\left\|A_{\theta}^{1 / 2} D_{N, \theta}^{1 / 2} \boldsymbol{w}\right\| ; \\
& \left\|\nabla A_{\theta}^{1 / 2} D_{N, \theta} \boldsymbol{w}\right\| \leq(N+1)^{1 / 2}\left\|\nabla A_{\theta}^{1 / 2} D_{N, \theta}^{1 / 2} \boldsymbol{w}\right\| ; \\
& \left\|\nabla A_{\theta} D_{N, \theta} \boldsymbol{w}\right\| \leq(N+1)^{1 / 2}\left\|\nabla A_{\theta} D_{N, \theta}^{1 / 2} \boldsymbol{w}\right\| .
\end{aligned}
$$

In particular estimate (17) is estimate (15) with $s=0$, while (18)-(20) come from (16) taking $p=1 / 2$ and replacing $\boldsymbol{w}$ by $A_{\theta}^{1 / 2} D_{N, \theta}^{1 / 2} \boldsymbol{w}, \nabla A_{\theta}^{1 / 2} D_{N, \theta}^{1 / 2} \boldsymbol{w}$ and $\nabla A_{\theta} D_{N, \theta}^{1 / 2} \boldsymbol{w}$ respectively. 


\section{A priori estimates}

In this section, we collect suitable a priori estimates for the weak solution to the problem (3)-(5), that will be used from Sect. 4 on to prove the existence of the global attractor.

We set $\lambda_{1}:=L^{-2}$ (first eigenvalue for the Laplace operator $-\Delta$ ), $\Lambda^{\theta}:=$ $(-\Delta)^{\theta / 2}$ with domain $\mathbf{H}^{\theta}$ and periodic boundary conditions $\left(\Lambda^{\theta}\right.$ is a positive self-adjoint operator in $\left.\mathbf{L}^{2}\right), \Lambda:=\Lambda^{1}$, and

$$
\begin{aligned}
k_{0} & :=\left\|A_{\theta}^{1 / 2} D_{N, \theta}^{1 / 2} \boldsymbol{w}(0)\right\|^{2}, \\
K_{1} & :=\frac{\left\|\Lambda^{-1} A_{\theta}^{-1 / 2} D_{N, \theta}^{1 / 2} \boldsymbol{f}\right\|^{2}}{\nu}, \\
k_{1} & :=k_{0}+\frac{K_{1}}{\nu \lambda_{1}} .
\end{aligned}
$$

Theorem 3.1. If $\boldsymbol{w}$ is a regular weak solution of (3)-(5), for arbitrary $t \geq$ $0, r>0$ we have

$$
\begin{aligned}
& \left\|A_{\theta}^{1 / 2} D_{N, \theta}^{1 / 2} \boldsymbol{w}(t)\right\|^{2} \leq k_{0} \mathrm{e}^{-\nu \lambda_{1} t}+\frac{K_{1}}{\nu \lambda_{1}}\left(1-\mathrm{e}^{-\nu \lambda_{1} t}\right) \leq k_{1}, \\
& \int_{t}^{t+r} \nu\left\|\nabla A_{\theta}^{1 / 2} D_{N, \theta}^{1 / 2} \boldsymbol{w}(s)\right\|^{2} \mathrm{~d} s \leq r K_{1}+k_{1}, \\
& \int_{0}^{t} \mathrm{e}^{-\nu \lambda_{1} s / C} \nu\left\|\nabla A_{\theta}^{1 / 2} D_{N, \theta}^{1 / 2} \boldsymbol{w}(s)\right\|^{2} \mathrm{~d} s \leq \frac{C K_{1}}{\nu \lambda_{1}}+k_{0}, \quad \forall C>0 \text { given. }
\end{aligned}
$$

Remark 3.2. (i) From (24) we get that

$$
t \mapsto\left\|A_{\theta}^{1 / 2} D_{N, \theta}^{1 / 2} \boldsymbol{w}(t)\right\| \in \mathrm{L}^{\infty}(0,+\infty) .
$$

Then, in view of (12), we obtain that

$$
D_{N, \theta}^{1 / 2} \boldsymbol{w} \in \mathrm{L}^{\infty}\left(0,+\infty ; \mathbf{H}_{\sigma}^{\theta}\right)
$$

and, in view of Lemma 2.8 with $p=1 / 2$, we also deduce

$$
\boldsymbol{w} \in \mathrm{L}^{\infty}\left(0,+\infty ; \mathbf{H}_{\sigma}^{\theta}\right) .
$$

(ii) From (25) we find that

$$
t \mapsto\left\|\nabla A_{\theta}^{1 / 2} D_{N, \theta}^{1 / 2} \boldsymbol{w}(t)\right\| \in \mathrm{L}_{\mathrm{loc}}^{2}(0,+\infty),
$$

which yields, in view of Lemma 2.8 and (12),

$$
\boldsymbol{w} \in \mathrm{L}_{\mathrm{loc}}^{2}\left(0,+\infty ; \mathbf{H}_{\sigma}^{1+\theta}\right) .
$$

Proof. To be rigorous, in order to prove the estimates one should pass through a sequence of Galerkin approximating solutions of (3)-(5), then pass to the limit in the resulting estimates. In order to avoid overloading calculations, here, we derive the estimates by arguing directly, at a formal level, on the solution of the problem (3)-(5). We refer to [1] and [4] for a detailed discussion of the Galerkin approximation technique. 
Let us prove (24). We test the Eq. (3) against $A_{\theta} D_{N, \theta} \boldsymbol{w}$. Leibniz's rule and differentiation under the integral sign give

$$
\left(\partial_{t} \boldsymbol{w}, A_{\theta} D_{N, \theta} \boldsymbol{w}\right)=\left(\partial_{t} A_{\theta}^{1 / 2} D_{N, \theta}^{1 / 2} \boldsymbol{w}, A_{\theta}^{1 / 2} D_{N, \theta}^{1 / 2} \boldsymbol{w}\right)=\frac{1}{2} \frac{\mathrm{d}}{\mathrm{d} t}\left\|A_{\theta}^{1 / 2} D_{N, \theta}^{1 / 2} \boldsymbol{w}(t)\right\|^{2} .
$$

Since $\nabla \cdot \boldsymbol{w}=0$, by using (93) we get

$$
\left(\nabla \cdot\left(\overline{D_{N, \theta} \boldsymbol{w} \otimes D_{N, \theta} \boldsymbol{w}}\right), A_{\theta} D_{N, \theta} \boldsymbol{w}\right)=\left(\nabla \cdot\left(D_{N, \theta} \boldsymbol{w} \otimes D_{N, \theta} \boldsymbol{w}\right), D_{N, \theta} \boldsymbol{w}\right)=0 ;
$$

by using that the operators $A_{\theta}^{1 / 2}, D_{N, \theta}^{1 / 2}$ are self-adjoint, we get

$$
\begin{gathered}
-\nu\left(\Delta \boldsymbol{w}, A_{\theta} D_{N, \theta} \boldsymbol{w}\right)=-\nu\left(\nabla \cdot(\nabla \boldsymbol{w}), A_{\theta} D_{N, \theta} \boldsymbol{w}\right)=\nu\left(\nabla \boldsymbol{w}, \nabla A_{\theta} D_{N, \theta} \boldsymbol{w}\right) \\
=\nu\left(\nabla A_{\theta}^{1 / 2} D_{N, \theta}^{1 / 2} \boldsymbol{w}, \nabla A_{\theta}^{1 / 2} D_{N, \theta}^{1 / 2} \boldsymbol{w}\right)=\nu\left\|\nabla A_{\theta}^{1 / 2} D_{N, \theta}^{1 / 2} \boldsymbol{w}\right\|^{2} ;
\end{gathered}
$$

by using $\nabla \cdot \boldsymbol{w}=0$ and integration by parts, we get

$$
\left(\nabla q, A_{\theta} D_{N, \theta} \boldsymbol{w}\right)=-\left(q, A_{\theta} D_{N, \theta}(\nabla \cdot \boldsymbol{w})\right)=0 ;
$$

moreover

$$
\left(\overline{\boldsymbol{f}}, A_{\theta} D_{N, \theta} \boldsymbol{w}\right)=\left(\boldsymbol{f}, D_{N, \theta} \boldsymbol{w}\right)=\left(D_{N, \theta}^{1 / 2} \boldsymbol{f}, D_{N, \theta}^{1 / 2} \boldsymbol{w}\right) .
$$

Collecting the previous estimates we get

$$
\frac{1}{2} \frac{\mathrm{d}}{\mathrm{d} t}\left\|A_{\theta}^{1 / 2} D_{N, \theta}^{1 / 2} \boldsymbol{w}(t)\right\|^{2}+\nu\left\|\nabla A_{\theta}^{1 / 2} D_{N, \theta}^{1 / 2} \boldsymbol{w}(t)\right\|^{2}=\left(D_{N, \theta}^{1 / 2} \boldsymbol{f}, D_{N, \theta}^{1 / 2} \boldsymbol{w}(t)\right) .
$$

We estimate the right-hand side by $\left(\Lambda=(-\Delta)^{1 / 2}\right)$

$$
\begin{aligned}
\left|\left(D_{N, \theta}^{1 / 2} \boldsymbol{f}, D_{N, \theta}^{1 / 2} \boldsymbol{w}\right)\right| & =\left|\left(\Lambda^{-1} A_{\theta}^{-1 / 2} D_{N, \theta}^{1 / 2} \boldsymbol{f}, \Lambda A_{\theta}^{1 / 2} D_{N, \theta}^{1 / 2} \boldsymbol{w}\right)\right| \\
& \leq\left\|\Lambda^{-1} A_{\theta}^{-1 / 2} D_{N, \theta}^{1 / 2} \boldsymbol{f}\right\|\left\|\Lambda A_{\theta}^{1 / 2} D_{N, \theta}^{1 / 2} \boldsymbol{w}\right\| \\
& \leq \frac{\left\|\Lambda^{-1} A_{\theta}^{-1 / 2} D_{N, \theta}^{1 / 2} \boldsymbol{f}\right\|^{2}}{2 \nu}+\frac{\nu}{2}\left\|\Lambda A_{\theta}^{1 / 2} D_{N, \theta}^{1 / 2} \boldsymbol{w}\right\|^{2} \\
& =\frac{1}{2}\left\{K_{1}+\nu\left\|\nabla A_{\theta}^{1 / 2} D_{N, \theta}^{1 / 2} \boldsymbol{w}\right\|^{2}\right\}
\end{aligned}
$$

by Cauchy-Schwarz inequality and definition of $K_{1}$. We notice that in the estimate above we used that $\|\Lambda \boldsymbol{w}\|=\|\nabla \boldsymbol{w}\| .^{2}$

We deduce

$$
\frac{\mathrm{d}}{\mathrm{d} t}\left\|A_{\theta}^{1 / 2} D_{N, \theta}^{1 / 2} \boldsymbol{w}\right\|^{2}+\nu\left\|\nabla A_{\theta}^{1 / 2} D_{N, \theta}^{1 / 2} \boldsymbol{w}(t)\right\|^{2} \leq K_{1}
$$

and, thanks to the Poincaré inequality,

\footnotetext{
${ }^{2}$ For every $\boldsymbol{w}$ we compute by Parseval's identity

$\|\nabla \boldsymbol{w}\|^{2}=\sum_{j=1}^{3}\left\|\partial_{j} \boldsymbol{w}\right\|^{2}=\sum_{j=1}^{3} \sum_{\boldsymbol{k}}\left|\mathrm{i} k_{j} \widehat{\boldsymbol{w}}_{\boldsymbol{k}}\right|^{2}=\sum_{j=1}^{3} \sum_{\boldsymbol{k}}\left|k_{j}\right|^{2}\left|\widehat{\boldsymbol{w}}_{\boldsymbol{k}}\right|^{2}=\sum_{\boldsymbol{k}}|\boldsymbol{k}|^{2}\left|\widehat{\boldsymbol{w}}_{\boldsymbol{k}}\right|^{2}=\|\Lambda \boldsymbol{w}\|^{2}$.
} 


$$
\frac{\mathrm{d}}{\mathrm{d} t}\left\|A_{\theta}^{1 / 2} D_{N, \theta}^{1 / 2} \boldsymbol{w}(t)\right\|^{2}+\nu \lambda_{1}\left\|A_{\theta}^{1 / 2} D_{N, \theta}^{1 / 2} \boldsymbol{w}(t)\right\|^{2} \leq K_{1} ;
$$

an application of Gronwall's Lemma yields

$$
\left\|A_{\theta}^{1 / 2} D_{N, \theta}^{1 / 2} \boldsymbol{w}(t)\right\|^{2} \leq k_{0} \mathrm{e}^{-\nu \lambda_{1} t}+\frac{K_{1}}{\nu \lambda_{1}}\left(1-\mathrm{e}^{-\nu \lambda_{1} t}\right)
$$

which gives (24) and in particular

$$
\left\|A_{\theta}^{1 / 2} D_{N, \theta}^{1 / 2} \boldsymbol{w}(t)\right\|^{2} \leq k_{1},
$$

which implies (see Remark 3.2)

$$
\boldsymbol{w} \in \mathrm{L}^{\infty}\left(0, \infty ; \mathbf{H}_{\sigma}^{\theta}\right) .
$$

Let us prove now (25). Integrating (27) over $[t, t+r]$ and neglecting $\left\|A_{\theta}^{1 / 2} D_{N, \theta}^{1 / 2} \boldsymbol{w}(t+r)\right\|^{2}$ in the left-hand side, we get

$$
\int_{t}^{t+r} \nu\left\|\nabla A_{\theta}^{1 / 2} D_{N, \theta}^{1 / 2} \boldsymbol{w}(s)\right\|^{2} \mathrm{~d} s \leq r K_{1}+\left\|A_{\theta}^{1 / 2} D_{N, \theta}^{1 / 2} \boldsymbol{w}(t)\right\|^{2}
$$

and using (24) yields (25).

Let us prove (26). Now, let us multiply $(27)$ by $\mathrm{e}^{-\nu \lambda_{1} t / C}>0$ and integrate in time over the interval $[0, t]$ :

$$
\begin{gathered}
\int_{0}^{t} \mathrm{e}^{-\nu \lambda_{1} s / C} \frac{\mathrm{d}}{\mathrm{d} s}\left\|A_{\theta}^{1 / 2} D_{N, \theta}^{1 / 2} \boldsymbol{w}(s)\right\|^{2} \mathrm{~d} s+\int_{0}^{t} \mathrm{e}^{-\nu \lambda_{1} s / C} \nu\left\|\nabla A_{\theta}^{1 / 2} D_{N, \theta}^{1 / 2} \boldsymbol{w}(s)\right\|^{2} \mathrm{~d} t \\
\leqslant \int_{0}^{t} K_{1} \mathrm{e}^{-\nu \lambda_{1} s / C} \mathrm{~d} s=\frac{C K_{1}}{\nu \lambda_{1}}\left(1-\mathrm{e}^{-\nu \lambda_{1} t / C}\right) \leqslant \frac{C K_{1}}{\nu \lambda_{1}} .
\end{gathered}
$$

Integrating by parts, the first integral in the left-hand side of the above inequality becomes

$$
\left[\mathrm{e}^{-\nu \lambda_{1} s / C}\left\|A_{\theta}^{1 / 2} D_{N, \theta}^{1 / 2} \boldsymbol{w}(s)\right\|^{2}\right]_{0}^{t}+\frac{\nu \lambda_{1}}{C} \int_{0}^{t} \mathrm{e}^{-\nu \lambda_{1} s / C}\left\|A_{\theta}^{1 / 2} D_{N, \theta}^{1 / 2} \boldsymbol{w}(s)\right\|^{2} \mathrm{~d} s,
$$

hence we have

$$
\begin{aligned}
& \mathrm{e}^{-\nu \lambda_{1} t / C}\left\|A_{\theta}^{1 / 2} D_{N, \theta}^{1 / 2} \boldsymbol{w}(t)\right\|^{2}+\frac{\nu \lambda_{1}}{C} \int_{0}^{t} \mathrm{e}^{-\nu \lambda_{1} s / C}\left\|A_{\theta}^{1 / 2} D_{N, \theta}^{1 / 2} \boldsymbol{w}(s)\right\|^{2} \mathrm{~d} s \\
& \quad+\int_{0}^{t} \mathrm{e}^{-\nu \lambda_{1} s / C} \nu\left\|\nabla A_{\theta}^{1 / 2} D_{N, \theta}^{1 / 2} \boldsymbol{w}(s)\right\|^{2} \mathrm{~d} s \\
& \leqslant \frac{C K_{1}}{\nu \lambda_{1}}+k_{0}
\end{aligned}
$$

neglecting the first two terms, which are positive quantities, provides (26).

\section{Existence of the global attractor}

\subsection{Some basic definitions}

To make the exposition self-contained, we recall below some basic concepts and results on invariant sets and attractors in dynamical systems that will be used later on. 
Definition 4.1. Let $(W, d)$ be a metric space. A semigroup on $(W, d)$ is a family of operators $(S(t))_{t \geq 0}, S(t): W \rightarrow W$, such that

(i) $S(0) w=w$, for all $w \in W$;

(ii) $S(s) S(t) w=S(t+s) w$ for all $w \in W$ and for every $s, t \geq 0$.

A semigroup $(S(t))_{t \geq 0}$ is said to be continuous (or a semiflow) if

$$
S(t): W \rightarrow W
$$

is a continuous operator from $W$ to itself, for every $t \geq 0$.

Definition 4.2. Let $(S(t))_{t \geq 0}$ be a semigroup on $(W, d)$. We say that the operators $S(t)$ are uniformly compact for $t$ large if for every bounded set $\mathcal{B}$ there exists $t_{0}$, which may depend on $\mathcal{B}$, such that

$$
\bigcup_{t \geq t_{0}} S(t) \mathcal{B}
$$

is relatively compact in $W$.

Definition 4.3. We say that $\mathcal{A} \subset W$ is a global attractor for the semiflow if

(1) $\mathcal{A}$ is nonempty and compact;

(2) $S(t) \mathcal{A}=\mathcal{A}$ for all $t \geq 0$ (i.e. $\mathcal{A}$ is invariant);

(3) for all bounded sets $B \subset W$, we have

$$
\lim _{t \rightarrow+\infty} \delta(S(t) B, \mathcal{A})=0,
$$

where $\delta(X, Y):=\sup _{x \in X} \inf _{y \in Y} d(x, y)$ is the Hausdorff semidistance between the pair of sets $X, Y \subset W$.

In order to establish the existence of attractors, a useful concept is the related concept of absorbing set.

Definition 4.4. Let $\mathcal{B}$ be a subset of $W$ and $\mathcal{U}$ be an open set containing $\mathcal{B}$. We say that $\mathcal{B}$ is absorbing in $\mathcal{U}$ if

$\forall \mathcal{B}_{0} \subset \mathcal{U}, \quad \mathcal{B}_{0}$ bounded, $\exists t_{1}\left(\mathcal{B}_{0}\right)$ such that $S(t) \mathcal{B}_{0} \subset \mathcal{B}, \forall t \geq t_{1}$.

When $\mathcal{U}=W$ in the preceding definition, then we simply say that $\mathcal{B}$ is an absorbing set in $W$.

Remark 4.5. Let us observe that a global attractor for a semigroup $(S(t))_{t \geq 0}$, if it exists, is necessarily unique and coincides with the omega-limit of an absorbing set. We remind that the omega-limit of a set $\mathcal{B} \subset W$ is defined by

$$
\omega(\mathcal{B}):=\bigcap_{s \geq 0} \overline{\bigcup_{t \geq s} S(t) \mathcal{B}} .
$$

The following theorem on the existence of the global attractor is proven in [19] (see also [17]).

Theorem 4.6. Let $(W, d)$ be a metric space and $(S(t))_{t \geq 0}$ a continuous semigroup on $W$, such that $S(t)$ is uniformly compact for $t$ large. Assume moreover that there exists a bounded absorbing set $\mathcal{B} \subset W$. Then there exists the (unique) 
global attractor $\mathcal{A}$ for the semigroup $(S(t))_{t \geq 0}$, which is given by the omegalimit of the set $\mathcal{B}$, i.e.

$$
\mathcal{A}=\omega(\mathcal{B})=\bigcap_{s \geq 0} \overline{\bigcup_{t \geq s} S(t) \mathcal{B}}
$$

\subsection{Existence (proof of Theorem 1.1)}

In this section, the theoretical results collected above will be applied to the continuous semigroup $(S(t))_{t \geq 0}$ on $\mathbf{H}_{\sigma}^{\theta}$ defined by

$$
\begin{gathered}
\forall t \geq 0, \quad S(t): \mathbf{H}_{\sigma}^{\theta} \rightarrow \mathbf{H}_{\sigma}^{\theta} \\
\boldsymbol{w}_{0} \mapsto S(t) \boldsymbol{w}_{0}:=\boldsymbol{w}(t, \cdot),
\end{gathered}
$$

where $\boldsymbol{w}=\boldsymbol{w}(t, \cdot)$ is the regular weak solution of the problem (3)-(5) with initial data $\boldsymbol{w}_{0}$.

We notice that the continuity of the operators $S(t)$ in $(28)$ directly follows from the definition of regular weak solution, see Sect. 2.2.

In order to prove the existence of the global attractor for the semigroup (28), as an application of Theorem 4.6, we need the following lemmas. Observe that, in this section, we provide the spaces $\mathbf{H}_{\sigma}^{\theta}$ and $\mathbf{H}_{\sigma}^{2 \theta}$ with the norms

$$
\|\boldsymbol{w}\|_{\mathbf{H}^{\theta}}:=\left\|A_{\theta}^{1 / 2} D_{N, \theta}^{1 / 2} \boldsymbol{w}\right\|, \quad\|\boldsymbol{w}\|_{\mathbf{H}^{2 \theta}}:=\left\|A_{\theta} D_{N, \theta}^{1 / 2} \boldsymbol{w}\right\|,
$$

which are respectively equivalent to the norms $\|\boldsymbol{w}\|_{\theta, 2}$ and $\|\boldsymbol{w}\|_{2 \theta, 2}$ [see Remark 2.4 and (16)].

Lemma 4.7. If $\theta \geq 1 / 2$ and $\mathcal{B}_{0}$ is a bounded subset of $\mathbf{H}_{\sigma}^{\theta}$, then there exists $t^{\prime}=t^{\prime}\left(\mathcal{B}_{0}\right)>0$ such that, if $\boldsymbol{w}$ is a regular weak solution of $(3)-(5)$ with arbitrary initial datum $\boldsymbol{w}_{0} \in \mathcal{B}_{0}$, then

$$
\left\|A_{\theta}^{1 / 2} D_{N, \theta}^{1 / 2} \boldsymbol{w}(t)\right\|^{2} \leq \frac{2 K_{1}}{\nu \lambda_{1}}, \quad \forall t \geq t^{\prime},
$$

where $K_{1}$ is defined in (22) and $\lambda_{1}$ is the first eigenvalue of the Laplace operator $-\Delta$. In particular, we have

$$
\limsup _{t \rightarrow+\infty}\left\|A_{\theta}^{1 / 2} D_{N, \theta}^{1 / 2} \boldsymbol{w}(t)\right\|^{2} \leq \frac{2 K_{1}}{\nu \lambda_{1}}=: r_{1}^{2} .
$$

Notice that the constant $r_{1}$ is independent of the initial datum.

Proof. The estimate (29) follows at once from the estimate (24) by noticing that the right-hand side of the latter estimate

$$
g(t):=k_{0} \mathrm{e}^{-\nu \lambda_{1} t}+\frac{K_{1}}{\nu \lambda_{1}}\left(1-\mathrm{e}^{-\nu \lambda_{1} t}\right) \leq R^{2} \mathrm{e}^{-\nu \lambda_{1} t}+\frac{K_{1}}{\nu \lambda_{1}}\left(1-\mathrm{e}^{-\nu \lambda_{1} t}\right)
$$

tends to $\frac{K_{1}}{\nu \lambda_{1}}$ as $t \rightarrow+\infty$, where $R=\sup _{\boldsymbol{w}_{0} \in \mathcal{B}_{0}}\left\|A_{\theta}^{1 / 2} D_{N, \theta}^{1 / 2} \boldsymbol{w}_{0}\right\|$; thus there exists $t^{\prime}>0$ such that $g(t) \leq \frac{2 K_{1}}{\nu \lambda_{1}}$ for $t \geq t^{\prime}$.

Remark 4.8. As a consequence of Lemma 4.7, see also (15), we obtain that the ball

$$
\mathcal{B}_{1}=\mathcal{B}_{1}\left(0 ; r_{1}\right):=\left\{\boldsymbol{w} \in \mathbf{H}_{\sigma}^{\theta}:\left\|A_{\theta}^{1 / 2} D_{N, \theta}^{1 / 2} \boldsymbol{w}\right\| \leq r_{1}\right\}
$$


is a bounded absorbing set in $\mathbf{H}_{\sigma}^{\theta}$; indeed by Lemma 4.7 we get that for every bounded set $\mathcal{B}_{0} \subset \mathbf{H}_{\sigma}^{\theta}$ we have

$$
S(t) \mathcal{B}_{0} \subset \mathcal{B}_{1}, \quad \forall t \geq t^{\prime},
$$

being $t^{\prime}>0$ the time given in Lemma 4.7 .

Lemma 4.9. Assume that $\boldsymbol{f} \in \boldsymbol{H}^{-1}, \theta \geq 1 / 2$ and let $\mathcal{B}_{0}$ be a bounded subset of $\mathbf{H}_{\sigma}^{\theta}$. There exist $r_{2}=r_{2}\left(\nu, N, \theta, K_{1}, \lambda_{1}\right)>0$ and a time $t^{\prime \prime}=t^{\prime \prime}\left(\mathcal{B}_{0}\right)>t^{\prime}$ such that, if $\boldsymbol{w}$ is the regular weak solution of (3)-(5) with arbitrary initial datum $\boldsymbol{w}_{0} \in \mathcal{B}_{0}$, then

$$
\left\|A_{\theta} D_{N, \theta}^{1 / 2} \boldsymbol{w}(t)\right\|^{2} \leq r_{2}^{2}, \quad \forall t \geq t^{\prime \prime} .
$$

In particular, we have

$$
\limsup _{t \rightarrow+\infty}\left\|A_{\theta} D_{N, \theta}^{1 / 2} \boldsymbol{w}(t)\right\|^{2} \leq r_{2}^{2}
$$

Proof. We use $A_{\theta}^{2} D_{N, \theta} \boldsymbol{w}$ as test function for the Eq. (3) (now formally, but the procedure actually goes through the Galerkin approximation).

Thus, testing the Eq. (3) against $A_{\theta}^{2} D_{N, \theta} \boldsymbol{w}$, and arguing as in the proof of Theorem 3.1 we get

$$
\begin{aligned}
& \left(\partial_{t} \boldsymbol{w}, A_{\theta}^{2} D_{N, \theta} \boldsymbol{w}\right)=\frac{1}{2} \partial_{t}\left(A_{\theta} D_{N, \theta}^{1 / 2} \boldsymbol{w}, A_{\theta} D_{N, \theta}^{1 / 2} \boldsymbol{w}\right)=\frac{1}{2} \frac{\mathrm{d}}{\mathrm{d} t}\left\|A_{\theta} D_{N, \theta}^{1 / 2} \boldsymbol{w}\right\|^{2} ; \\
& \left(\nabla \cdot\left(\overline{D_{N, \theta} \boldsymbol{w} \otimes D_{N, \theta} \boldsymbol{w}}\right), A_{\theta}^{2} D_{N, \theta} \boldsymbol{w}\right)=\left(\nabla \cdot\left(D_{N, \theta} \boldsymbol{w} \otimes D_{N, \theta} \boldsymbol{w}\right), A_{\theta} D_{N, \theta} \boldsymbol{w}\right) ;
\end{aligned}
$$

using that $-\Delta=\Lambda^{2}$ and $\nabla \cdot \boldsymbol{w}=0$

$$
\begin{aligned}
& -\nu\left(\Delta \boldsymbol{w}, A_{\theta}^{2} D_{N, \theta} \boldsymbol{w}\right)=\nu\left(\Lambda^{2} \boldsymbol{w}, A_{\theta}^{2} D_{N, \theta} \boldsymbol{w}\right)=\nu\left(\Lambda A_{\theta} D_{N, \theta}^{1 / 2} \boldsymbol{w}, \Lambda A_{\theta} D_{N, \theta}^{1 / 2} \boldsymbol{w}\right) \\
& =\nu\left\|\Lambda A_{\theta} D_{N, \theta}^{1 / 2} \boldsymbol{w}\right\|^{2}=\nu\left\|\nabla A_{\theta} D_{N, \theta}^{1 / 2} \boldsymbol{w}\right\|^{2} ; \\
& \left(\nabla q, A_{\theta}^{2} D_{N, \theta} \boldsymbol{w}\right)=-\left(q, A_{\theta}^{2} D_{N, \theta}(\nabla \cdot \boldsymbol{w})\right)=0 .
\end{aligned}
$$

Finally, we observe that

$$
\begin{aligned}
& \left(\overline{\boldsymbol{f}}, A_{\theta}^{2} D_{N, \theta} \boldsymbol{w}\right)=\left(\boldsymbol{f}, A_{\theta} D_{N, \theta} \boldsymbol{w}\right) \\
& \quad=\left(D_{N, \theta}^{1 / 2} \boldsymbol{f}, A_{\theta} D_{N, \theta}^{1 / 2} \boldsymbol{w}\right)=\left(\Lambda^{-1} D_{N, \theta}^{1 / 2} \boldsymbol{f}, \Lambda A_{\theta} D_{N, \theta}^{1 / 2} \boldsymbol{w}\right) \\
& \leq\left\|\Lambda^{-1} D_{N, \theta}^{1 / 2} \boldsymbol{f}\right\|\left\|\Lambda A_{\theta} D_{N, \theta}^{1 / 2} \boldsymbol{w}\right\|=\left\|\Lambda^{-1} D_{N, \theta}^{1 / 2} \boldsymbol{f}\right\|\left\|\nabla A_{\theta} D_{N, \theta}^{1 / 2} \boldsymbol{w}\right\| \\
& \quad \leq \frac{\left\|\Lambda^{-1} D_{N, \theta}^{1 / 2} \boldsymbol{f}\right\|^{2}}{\nu}+\frac{\nu}{4}\left\|\nabla A_{\theta} D_{N, \theta}^{1 / 2} \boldsymbol{w}\right\|^{2} .
\end{aligned}
$$

Collecting the previous inequalities we get

$$
\begin{aligned}
\frac{1}{2} & \frac{\mathrm{d}}{\mathrm{d} t}\left\|A_{\theta} D_{N, \theta}^{1 / 2} \boldsymbol{w}\right\|^{2}+\nu\left\|\nabla A_{\theta} D_{N, \theta}^{1 / 2} \boldsymbol{w}\right\|^{2} \\
= & \left(D_{N, \theta}^{1 / 2} \boldsymbol{f}, A_{\theta} D_{N, \theta}^{1 / 2} \boldsymbol{w}\right)-\left(\nabla \cdot\left(D_{N, \theta} \boldsymbol{w} \otimes D_{N, \theta} \boldsymbol{w}\right), A_{\theta} D_{N, \theta} \boldsymbol{w}\right) \\
\leq & \frac{\left\|\Lambda^{-1} D_{N, \theta}^{1 / 2} \boldsymbol{f}\right\|^{2}}{\nu}+\frac{\nu}{4}\left\|\nabla A_{\theta} D_{N, \theta}^{1 / 2} \boldsymbol{w}\right\|^{2} \\
& +\left|\left(\nabla \cdot\left(D_{N, \theta} \boldsymbol{w} \otimes D_{N, \theta} \boldsymbol{w}\right), A_{\theta} D_{N, \theta} \boldsymbol{w}\right)\right|
\end{aligned}
$$


Now we are going to make an estimate of the nonlinear term $\left(\nabla \cdot\left(D_{N, \theta} \boldsymbol{w} \otimes\right.\right.$ $\left.\left.D_{N, \theta} \boldsymbol{w}\right), A_{\theta} D_{N, \theta} \boldsymbol{w}\right)$ in the right-hand side of the above inequality. We firstly make use of the formula (91) (recall that $\nabla \cdot \boldsymbol{w}=0$ ) and Hölder's inequality to get

$$
\begin{aligned}
& \left|\left(\nabla \cdot\left(D_{N, \theta} \boldsymbol{w} \otimes D_{N, \theta} \boldsymbol{w}\right), A_{\theta} D_{N, \theta} \boldsymbol{w}\right)\right|=\left|\left(\left(D_{N, \theta} \boldsymbol{w} \cdot \nabla\right) D_{N, \theta} \boldsymbol{w}, A_{\theta} D_{N, \theta} \boldsymbol{w}\right)\right| \\
& \quad \leq\left\|D_{N, \theta} \boldsymbol{w}\right\|_{\mathrm{L}^{3}}\left\|\nabla D_{N, \theta} \boldsymbol{w}\right\|_{\mathrm{L}^{3}}\left\|A_{\theta} D_{N, \theta} \boldsymbol{w}\right\|_{\mathrm{L}^{3}} .
\end{aligned}
$$

Now we estimate each of the $\mathrm{L}^{3}$-factors appearing in the right-hand side of the inequality above as follows. to get

The estimate of $\left\|D_{N, \theta} \boldsymbol{w}\right\|_{\mathrm{L}^{3}}$. We apply (95) to the function $\boldsymbol{u}=D_{N, \theta} \boldsymbol{w}$

$$
\left\|D_{N, \theta} \boldsymbol{w}\right\|_{\mathrm{L}^{3}} \leq C\left\|A_{\theta}^{1 / 2} D_{N, \theta} \boldsymbol{w}\right\|^{a}\left\|D_{N, \theta} \boldsymbol{w}\right\|^{1-a} .
$$

The estimate of $\left\|\nabla D_{N, \theta} \boldsymbol{w}\right\|_{\mathrm{L}^{3}}$. We apply (96) to the function $\boldsymbol{u}=D_{N, \theta} \boldsymbol{w}$ to get

$$
\left\|\nabla D_{N, \theta} \boldsymbol{w}\right\|_{L^{3}} \leq C\left\|\nabla A_{\theta} D_{N, \theta} \boldsymbol{w}\right\|^{b}\left\|A_{\theta}^{1 / 2} D_{N, \theta} \boldsymbol{w}\right\|^{1-b} .
$$

The estimate of $\left\|A_{\theta} D_{N, \theta} \boldsymbol{w}\right\|_{\mathrm{L}^{3}}$. We apply (97) to the function $\boldsymbol{u}=D_{N, \theta} \boldsymbol{w}$ to get

$$
\left\|A_{\theta} D_{N, \theta} \boldsymbol{w}\right\|_{\mathrm{L}^{3}} \leq C\left\|\nabla A_{\theta} D_{N, \theta} \boldsymbol{w}\right\|^{c}\left\|A_{\theta}^{1 / 2} D_{N, \theta} \boldsymbol{w}\right\|^{1-c} .
$$

The exponents $a, b, c$ involved in the estimates (33)-(35) above are those calculated in (99).

Using (33)-(35), we get

$$
\begin{aligned}
\mid(\nabla & \left.\cdot\left(D_{N, \theta} \boldsymbol{w} \otimes D_{N, \theta} \boldsymbol{w}\right), A_{\theta} D_{N, \theta} \boldsymbol{w}\right) \mid \leq C\left\|A_{\theta}^{1 / 2} D_{N, \theta} \boldsymbol{w}\right\|^{a}\left\|D_{N, \theta} \boldsymbol{w}\right\|^{1-a} \\
& \times\left\|\nabla A_{\theta} D_{N, \theta} \boldsymbol{w}\right\|^{b}\left\|A_{\theta}^{1 / 2} D_{N, \theta} \boldsymbol{w}\right\|^{1-b}\left\|\nabla A_{\theta} D_{N, \theta} \boldsymbol{w}\right\|\left\|_{\theta}^{1 / 2} D_{N, \theta} \boldsymbol{w}\right\|^{1-c} \\
= & C\left\|D_{N, \theta} \boldsymbol{w}\right\|^{1-a}\left\|A_{\theta}^{1 / 2} D_{N, \theta} \boldsymbol{w}\right\|^{2-b-c+a}\left\|\nabla A_{\theta} D_{N, \theta} \boldsymbol{w}\right\|^{b+c} .
\end{aligned}
$$

1. We estimate the factor involving $\left\|D_{N, \theta} \boldsymbol{w}\right\|$ by using (17) to get

$$
\left\|D_{N, \theta} \boldsymbol{w}\right\|^{1-a} \leq\left\|A_{\theta}^{1 / 2} D_{N, \theta}^{1 / 2} \boldsymbol{w}\right\|^{1-a}
$$

2. We estimate the factor involving $\left\|A_{\theta}^{1 / 2} D_{N, \theta} \boldsymbol{w}\right\|$ by using (18) to get

$$
\left\|A_{\theta}^{1 / 2} D_{N, \theta} \boldsymbol{w}\right\|^{2-b-c+a} \leq(N+1)^{\frac{2-b-c+a}{2}}\left\|A_{\theta}^{1 / 2} D_{N, \theta}^{1 / 2} \boldsymbol{w}\right\|^{2-b-c+a} .
$$

3. Finally, we estimate the factor involving $\left\|\nabla A_{\theta} D_{N, \theta} \boldsymbol{w}\right\|$ by using (20) to get

$$
\left\|\nabla A_{\theta} D_{N, \theta} \boldsymbol{w}\right\|^{b+c} \leq(N+1)^{\frac{b+c}{2}}\left\|\nabla A_{\theta} D_{N, \theta}^{1 / 2} \boldsymbol{w}\right\|^{b+c} .
$$

From (36)-(39) we get 


$$
\begin{aligned}
\mid & \left(\nabla \cdot\left(D_{N, \theta} \boldsymbol{w} \otimes D_{N, \theta} \boldsymbol{w}\right), A_{\theta} D_{N, \theta} \boldsymbol{w}\right) \mid \\
& \leq C(N+1)^{1+\frac{a}{2}}\left\|A_{\theta}^{1 / 2} D_{N, \theta}^{1 / 2} \boldsymbol{w}\right\|^{3-b-c}\left\|\nabla A_{\theta} D_{N, \theta}^{1 / 2} \boldsymbol{w}\right\|^{b+c} \\
& \leq \frac{\nu}{4}\left\|\nabla A_{\theta} D_{N, \theta}^{1 / 2} \boldsymbol{w}\right\|^{2}+\frac{C}{\nu^{\frac{b+c}{2} p^{\prime}}}(N+1)^{\left(1+\frac{a}{2}\right) p^{\prime}}\left\|A_{\theta}^{1 / 2} D_{N, \theta}^{1 / 2} \boldsymbol{w}\right\|^{(3-b-c) p^{\prime}} \\
& =\frac{\nu}{4}\left\|\nabla A_{\theta} D_{N, \theta}^{1 / 2} \boldsymbol{w}\right\|^{2}+\frac{C}{\nu^{\frac{1}{\theta}}}(N+1)^{\left(1+\frac{1}{4 \theta}\right) \frac{\theta+1}{\theta}}\left\|A_{\theta}^{1 / 2} D_{N, \theta}^{1 / 2} \boldsymbol{w}\right\|^{3+\frac{1}{\theta}},
\end{aligned}
$$

where Young's inequality has been used with $p=\frac{2}{b+c}=\theta+1$ and $p^{\prime}=\frac{2}{2-b-c}=$ $\frac{\theta+1}{\theta}$ is the conjugate exponent of $p$ [note that $p>1$, as $b+c<2$ thanks to (101)] and where we have computed [see (99)]

$$
\frac{b+c}{2} p^{\prime}=\frac{1}{\theta}, \quad\left(1+\frac{a}{2}\right) p^{\prime}=\left(1+\frac{1}{4 \theta}\right) \frac{\theta+1}{\theta}, \quad(3-b-c) p^{\prime}=3+\frac{1}{\theta} .
$$

Coming back to (32) and using (40) to estimate the nonlinear term in the right-hand side, we obtain

$$
\begin{aligned}
\frac{1}{2} \frac{\mathrm{d}}{\mathrm{d} t}\left\|A_{\theta} D_{N, \theta}^{1 / 2} \boldsymbol{w}\right\|^{2}+\nu\left\|\nabla A_{\theta} D_{N, \theta}^{1 / 2} \boldsymbol{w}\right\|^{2} \\
\leq \frac{\left\|\Lambda^{-1} D_{N, \theta}^{1 / 2} \boldsymbol{f}\right\|^{2}}{\nu}+\frac{\nu}{2}\left\|\nabla A_{\theta} D_{N, \theta}^{1 / 2} \boldsymbol{w}\right\|^{2} \\
\quad+\frac{C}{\nu^{\frac{1}{\theta}}}(N+1)^{\left(1+\frac{1}{4 \theta}\right) \frac{\theta+1}{\theta}}\left\|A_{\theta}^{1 / 2} D_{N, \theta}^{1 / 2} \boldsymbol{w}\right\|^{3+\frac{1}{\theta}} .
\end{aligned}
$$

Absorbing on the left the term $\frac{\nu}{2}\left\|\nabla A_{\theta} D_{N, \theta}^{1 / 2} \boldsymbol{w}\right\|^{2}$ appearing in the right-hand side of the estimate above, estimating $\left\|A_{\theta}^{1 / 2} D_{N, \theta}^{1 / 2} \boldsymbol{w}\right\|^{3+1 / \theta}$ by (29) we then get

$$
\begin{aligned}
& \frac{\mathrm{d}}{\mathrm{d} t}\left\|A_{\theta} D_{N, \theta}^{1 / 2} \boldsymbol{w}\right\|^{2}+\nu\left\|\nabla A_{\theta} D_{N, \theta}^{1 / 2} \boldsymbol{w}\right\|^{2} \\
& \quad \leq 2 \frac{\left\|\Lambda^{-1} D_{N, \theta}^{1 / 2} \boldsymbol{f}\right\|^{2}}{\nu}+\frac{C}{\nu^{\frac{1}{\theta}}}(N+1)^{\left(1+\frac{1}{4 \theta}\right) \frac{\theta+1}{\theta}}\left(\frac{2 K_{1}}{\nu \lambda_{1}}\right)^{\frac{1}{2}\left(3+\frac{1}{\theta}\right)}=: K_{2}, \quad \forall t \geq t^{\prime} .
\end{aligned}
$$

Finally, using Poincaré's inequality

$$
\lambda_{1}\left\|A_{\theta} D_{N, \theta}^{1 / 2} \boldsymbol{w}\right\|^{2} \leq\left\|\nabla A_{\theta} D_{N, \theta}^{1 / 2} \boldsymbol{w}\right\|^{2}
$$

we get

$$
\frac{\mathrm{d}}{\mathrm{d} t}\left\|A_{\theta} D_{N, \theta}^{1 / 2} \boldsymbol{w}\right\|^{2}+\nu \lambda_{1}\left\|A_{\theta} D_{N, \theta}^{1 / 2} \boldsymbol{w}\right\|^{2} \leq K_{2}, \quad \forall t \geq t^{\prime}
$$

Then setting $y(t):=\left\|A_{\theta} D_{N, \theta}^{1 / 2} \boldsymbol{w}(t)\right\|^{2},(42)$ becomes

$$
y^{\prime}(t)+\nu \lambda_{1} y(t) \leq K_{2}, \quad \forall t \geq t^{\prime} .
$$

Multiplying (43) by $\mathrm{e}^{\nu \lambda_{1} t}$ then gives

$$
\frac{\mathrm{d}}{\mathrm{d} t}\left(\mathrm{e}^{\nu \lambda_{1} t} y(t)\right)=\mathrm{e}^{\nu \lambda_{1} t} y^{\prime}(t)+\nu \lambda_{1} \mathrm{e}^{\nu \lambda_{1} t} y(t) \leq K_{2} \mathrm{e}^{\nu \lambda_{1} t}, \quad \forall t \geq t^{\prime},
$$

hence integrating in time over $\left(t^{\prime}, t\right)$ for an arbitrary $t>t^{\prime}$

$$
y(t) \leq \mathrm{e}^{-\nu \lambda_{1}\left(t-t^{\prime}\right)} y\left(t^{\prime}\right)+\frac{K_{2}}{\nu \lambda_{1}}\left(1-\mathrm{e}^{-\nu \lambda_{1}\left(t-t^{\prime}\right)}\right), \quad \forall t \geq t^{\prime} .
$$


Let us observe that the value $K_{2}$ introduced in (41) does not depend on the initial data in (5). Since the right-hand side of (44) tends to $\frac{K_{2}}{\nu \lambda_{1}}$ as $t \rightarrow+\infty$, one can find a time $t^{\prime \prime}>t^{\prime}$ such that

$$
\left\|A_{\theta} D_{N, \theta}^{1 / 2} \boldsymbol{w}(t)\right\|^{2}=y(t) \leq \frac{2 K_{2}}{\nu \lambda_{1}}, \quad \forall t \geq t^{\prime \prime} .
$$

This just provides an estimate of type (31), where we take $r_{2}^{2}:=\frac{2 K_{2}}{\nu \lambda_{1}}$ (independent of the initial data). This ends the proof.

Remark 4.10. The result of Lemma 4.9 [see estimate (31)] tells that, as long as $\theta \geq 1 / 2$, a regular weak solution $\boldsymbol{w}$ to problem (3)-(5) with arbitrary initial datum $\boldsymbol{w}_{0} \in \mathbf{H}_{\sigma}^{\theta}$ gains some additional regularity, definitely in time, with respect to the one originally required at the beginning of Sect. 2.2: from the time $t^{\prime \prime}>0$ on, the solution $\boldsymbol{w}$ belongs to $\mathbf{H}_{\sigma}^{2 \theta}$ pointwise in time. Notice that, as a direct consequence of the definition of regular weak solution, we only deduce that $\boldsymbol{w} \in L_{\text {loc }}^{2}\left(0,+\infty ; \mathbf{H}_{\sigma}^{2 \theta}\right)$ (observing that $2 \theta \leq \theta+1$ as long as $\theta \leq 1)$

Let us denote by $\mathcal{B}_{2}$ the ball in $\mathbf{H}_{\sigma}^{2 \theta}$ :

$$
\mathcal{B}_{2}=\mathcal{B}_{2}\left(0 ; r_{2}\right):=\left\{\boldsymbol{w} \in \mathbf{H}_{\sigma}^{2 \theta}:\left\|A_{\theta} D_{N, \theta}^{1 / 2} \boldsymbol{w}\right\| \leq r_{2}\right\}
$$

As a consequence of Lemma 4.9, see also the norm equivalence in (13) and (16) (with $p=1 / 2$ ), we deduce that for every bounded set $\mathcal{B} \subset \mathbf{H}_{\sigma}^{\theta}$

$$
\bigcup_{t \geq t^{\prime \prime}} S(t) \mathcal{B} \subset \mathcal{B}_{2}
$$

being $t^{\prime \prime}=t^{\prime \prime}(\mathcal{B})>0$ the time given in Lemma 4.9.

Since $\mathbf{H}_{\sigma}^{2 \theta}$ is compactly imbedded in $\mathbf{H}_{\sigma}^{\theta}$, the ball $\mathcal{B}_{2}$ is a relatively compact subset of $\mathbf{H}_{\sigma}^{\theta}$. Hence for every bounded set $\mathcal{B} \subset \mathbf{H}_{\sigma}^{\theta}$

$$
\bigcup_{t \geq t^{\prime \prime}} S(t) \mathcal{B}
$$

is a relatively compact subset of $\mathbf{H}_{\sigma}^{\theta}$, which means, according to Definition 4.2 , that the operators $S(t)$ are uniformly compact for large $t$.

In view of the results collected in the previous Lemmas 4.7, 4.9, and the Remarks 4.8, 4.10, we may conclude that the semigroup $(S(t))_{t \geq 0}$ of operators defined in (28) enjoys all the assumptions required in Theorem 4.6. Hence applying the result of that theorem, we deduce that a unique global attractor $\mathcal{A}$ exists and is given by the omega-limit of the absorbing ball $\mathcal{B}_{1}$ in (30), namely

$$
\mathcal{A}=\omega\left(\mathcal{B}_{1}\right)
$$

We have thus proved Theorem 1.1. 


\section{Differentiability}

In this section we study the differentiability of the semigroup $S(t): \mathbf{H}_{\sigma}^{\theta} \rightarrow \mathbf{H}_{\sigma}^{\theta}$, given by $S(t) \boldsymbol{w}_{0}=\boldsymbol{w}(t, \cdot)$ [see (28)], with respect to the initial datum $\boldsymbol{w}_{0}$. The reason is that, in the subsequent section, we will investigate the Hausdorff and fractal dimensions of the global attractor $\mathcal{A}$ : the differentiability of the semigroup $S(t)$ is a sufficient condition in order to prove that the Hausdorff dimension of the attractor $\mathcal{A}$ is finite and to find an appropriate estimate of its value (see [19, Theorem 3.1]).

Consider the following systems of equations:

$$
\begin{aligned}
& \partial_{t} \boldsymbol{w}-\nu \Delta \boldsymbol{w}+\nabla q=\overline{\boldsymbol{f}}-\nabla \cdot \overline{D_{N, \theta} \boldsymbol{w} \otimes D_{N, \theta} \boldsymbol{w}} \\
& \nabla \cdot \boldsymbol{w}=0, \quad \boldsymbol{w}(0)=\boldsymbol{w}_{0} \\
& \partial_{t} \boldsymbol{v}-\nu \Delta \boldsymbol{v}+\nabla q_{1}=\overline{\boldsymbol{f}}-\nabla \cdot \overline{D_{N, \theta} \boldsymbol{v} \otimes D_{N, \theta} \boldsymbol{v}} \\
& \nabla \cdot \boldsymbol{v}=0, \quad \boldsymbol{v}(0)=\boldsymbol{v}_{0} \\
& \partial_{t} \boldsymbol{W}-\nu \Delta \boldsymbol{W}+\nabla Q=\overline{-\left(D_{N, \theta} \boldsymbol{W} \cdot \nabla\right) D_{N, \theta} \boldsymbol{w}-\left(D_{N, \theta} \boldsymbol{w} \cdot \nabla\right) D_{N, \theta} \boldsymbol{W}} \\
& \nabla \cdot \boldsymbol{W}=0, \quad \boldsymbol{W}(0)=\boldsymbol{w}_{0}-\boldsymbol{v}_{0},
\end{aligned}
$$

and set $\boldsymbol{\eta}:=\boldsymbol{w}-\boldsymbol{v}-\boldsymbol{W}$. In order to prove the Fréchet differentiability with respect to the initial datum of $S(t): \mathbf{H}_{\sigma}^{\theta} \rightarrow \mathbf{H}_{\sigma}^{\theta}$, it is sufficient to prove that

$$
\left\|A_{\theta}^{1 / 2} D_{N, \theta}^{1 / 2} \boldsymbol{\eta}(t)\right\| \leq C\left\|A_{\theta}^{1 / 2} D_{N, \theta}^{1 / 2}\left(\boldsymbol{w}_{0}-\boldsymbol{v}_{0}\right)\right\|^{2}
$$

for each $t \in[0, T)$.

Lemma 5.1. Assume $\theta \geq 1 / 2$. Given $T>0$ and defined $\boldsymbol{V}:=\boldsymbol{w}-\boldsymbol{v}$, there exists $\tilde{C}=\tilde{C}\left(T, N, \theta, K_{1}\right)>0$ such that

$$
\left\|A_{\theta}^{1 / 2} D_{N, \theta}^{1 / 2} \boldsymbol{V}(t)\right\|^{2}+\nu \int_{0}^{t}\left\|\nabla A_{\theta}^{1 / 2} D_{N, \theta}^{1 / 2} \boldsymbol{V}(s)\right\|^{2} d s \leq \tilde{C}\left\|A_{\theta}^{1 / 2} D_{N, \theta}^{1 / 2} \boldsymbol{V}(0)\right\|^{2}
$$

for each $t \in[0, T)$.

Proof. The equations satisfied by $\boldsymbol{V}$ are

$$
\begin{aligned}
& \partial_{t} \boldsymbol{V}-\nu \Delta \boldsymbol{V}+\nabla\left(q-q_{1}\right)=\overline{\left(D_{N, \theta} \boldsymbol{v} \cdot \nabla\right) D_{N, \theta} \boldsymbol{v}-\left(D_{N, \theta} \boldsymbol{w} \cdot \nabla\right) D_{N, \theta} \boldsymbol{w}} \\
& \quad=\overline{\left(D_{N, \theta} \boldsymbol{V} \cdot \nabla\right) D_{N, \theta} \boldsymbol{V}-\left(D_{N, \theta} \boldsymbol{V} \cdot \nabla\right) D_{N, \theta} \boldsymbol{w}-\left(D_{N, \theta} \boldsymbol{w} \cdot \nabla\right) D_{N, \theta} \boldsymbol{V}} \\
& \nabla \cdot \boldsymbol{V}=0, \quad \boldsymbol{V}(0)=\boldsymbol{w}_{0}-\boldsymbol{v}_{0} .
\end{aligned}
$$

Testing (47) by $A_{\theta} D_{N, \theta} \boldsymbol{V}$ and, recalling that $((\boldsymbol{u} \cdot \nabla) \boldsymbol{V}, \boldsymbol{V})=0$ provided that $\nabla \cdot \boldsymbol{u}=0$, we obtain [see formula (92) in Lemma A.1]

$$
\begin{aligned}
& \frac{1}{2} \frac{\mathrm{d}}{\mathrm{d} t}\left\|A_{\theta}^{1 / 2} D_{N, \theta}^{1 / 2} \boldsymbol{V}\right\|^{2}+\nu\left\|\nabla A_{\theta}^{1 / 2} D_{N, \theta}^{1 / 2} \boldsymbol{V}\right\|^{2}=-\left(\left(D_{N, \theta} \boldsymbol{V} \cdot \nabla\right) D_{N, \theta} \boldsymbol{w}, D_{N, \theta} \boldsymbol{V}\right) \\
& \quad=\left(\left(D_{N, \theta} \boldsymbol{V} \cdot \nabla\right) D_{N, \theta} \boldsymbol{V}, D_{N, \theta} \boldsymbol{w}\right) .
\end{aligned}
$$

We estimate

$$
\left|\left(\left(D_{N, \theta} \boldsymbol{V} \cdot \nabla\right) D_{N, \theta} \boldsymbol{V}, D_{N, \theta} \boldsymbol{w}\right)\right| \leq C\left\|D_{N, \theta} \boldsymbol{V}\right\|_{\mathrm{L}^{3}}\left\|\nabla D_{N, \theta} \boldsymbol{V}\right\|_{\mathrm{L}^{3}}\left\|D_{N, \theta} \boldsymbol{w}\right\|_{\mathrm{L}^{3}} .
$$


We estimate the $\mathrm{L}^{3}$-norms in (49) by using the inequality (95) to get, for $a=\frac{1}{2 \theta} \leq 1$,

$$
\begin{aligned}
& \left|\left(\left(D_{N, \theta} \boldsymbol{V} \cdot \nabla\right) D_{N, \theta} \boldsymbol{V}, D_{N, \theta} \boldsymbol{w}\right)\right| \\
& \leq \quad\left\|D_{N, \theta} \boldsymbol{V}\right\|^{1-a}\left\|A_{\theta}^{1 / 2} D_{N, \theta} \boldsymbol{V}\right\|^{a}\left\|\nabla D_{N, \theta} \boldsymbol{V}\right\|^{1-a}\left\|\nabla A_{\theta}^{1 / 2} D_{N, \theta} \boldsymbol{V}\right\|^{a} \\
& \quad \times\left\|D_{N, \theta} \boldsymbol{w}\right\|^{1-a}\left\|A_{\theta}^{1 / 2} D_{N, \theta} \boldsymbol{w}\right\|^{a} .
\end{aligned}
$$

To bound the factors involving the norms $\left\|D_{N, \theta} \boldsymbol{w}\right\|,\left\|D_{N, \theta} \boldsymbol{V}\right\|$ and $\left\|\nabla D_{N, \theta} \boldsymbol{V}\right\|$ in the right-hand side above, we apply the estimate (17) respectively to $\boldsymbol{w}, \boldsymbol{V}$ and $\nabla \boldsymbol{V}$; to bound the factors involving the norms $\left\|A_{\theta}^{1 / 2} D_{N, \theta} \boldsymbol{w}\right\|$, $\left\|A_{\theta}^{1 / 2} D_{N, \theta} \boldsymbol{V}\right\|$ and $\left\|\nabla A_{\theta}^{1 / 2} D_{N, \theta} \boldsymbol{V}\right\|$, we apply the estimates (18) and (19) to $\boldsymbol{w}$ and $\boldsymbol{V}$. Thus, from Young's inequality, we obtain

$$
\begin{aligned}
& \left|\left(\left(D_{N, \theta} \boldsymbol{V} \cdot \nabla\right) D_{N, \theta} \boldsymbol{V}, D_{N, \theta} \boldsymbol{w}\right)\right| \\
& \quad \leq C(N+1)^{\frac{3}{2} a}\left\|A_{\theta}^{1 / 2} D_{N, \theta}^{1 / 2} \boldsymbol{V}\right\|\left\|\nabla A_{\theta}^{1 / 2} D_{N, \theta}^{1 / 2} \boldsymbol{V}\right\|\left\|A_{\theta}^{1 / 2} D_{N, \theta}^{1 / 2} \boldsymbol{w}\right\| \\
& \quad \leq \frac{\nu}{2}\left\|\nabla A_{\theta}^{1 / 2} D_{N, \theta}^{1 / 2} \boldsymbol{V}\right\|^{2}+\frac{C}{\nu}(N+1)^{3 a}\left\|A_{\theta}^{1 / 2} D_{N, \theta}^{1 / 2} \boldsymbol{w}\right\|^{2}\left\|A_{\theta}^{1 / 2} D_{N, \theta}^{1 / 2} \boldsymbol{V}\right\|^{2} .
\end{aligned}
$$

Coming back to (48) and using (50) we get

$$
\begin{aligned}
& \frac{1}{2} \frac{\mathrm{d}}{\mathrm{d} t}\left\|A_{\theta}^{1 / 2} D_{N, \theta}^{1 / 2} \boldsymbol{V}\right\|^{2}+\nu\left\|\nabla A_{\theta}^{1 / 2} D_{N, \theta}^{1 / 2} \boldsymbol{V}\right\|^{2} \\
& \quad \leq \frac{\nu}{2}\left\|\nabla A_{\theta}^{1 / 2} D_{N, \theta}^{1 / 2} \boldsymbol{V}\right\|^{2}+\frac{C}{\nu}(N+1)^{3 a}\left\|A_{\theta}^{1 / 2} D_{N, \theta}^{1 / 2} \boldsymbol{w}\right\|^{2}\left\|A_{\theta}^{1 / 2} D_{N, \theta}^{1 / 2} \boldsymbol{V}\right\|^{2}
\end{aligned}
$$

Absorbing in the left-hand side the term $\frac{\nu}{2}\left\|\nabla A_{\theta}^{1 / 2} D_{N, \theta}^{1 / 2} \boldsymbol{V}\right\|^{2}$ we find

$$
\begin{aligned}
\frac{1}{2} & \frac{\mathrm{d}}{\mathrm{d} t}\left\|A_{\theta}^{1 / 2} D_{N, \theta}^{1 / 2} \boldsymbol{V}\right\|^{2}+\frac{\nu}{2}\left\|\nabla A_{\theta}^{1 / 2} D_{N, \theta}^{1 / 2} \boldsymbol{V}\right\|^{2} \\
& \leq \frac{C}{\nu}(N+1)^{3 a}\left\|A_{\theta}^{1 / 2} D_{N, \theta}^{1 / 2} \boldsymbol{w}\right\|^{2}\left\|A_{\theta}^{1 / 2} D_{N, \theta}^{1 / 2} \boldsymbol{V}\right\|^{2} \\
& \leq \frac{C}{\nu}(N+1)^{3 a}\left\|A_{\theta}^{1 / 2} D_{N, \theta}^{1 / 2} \boldsymbol{V}\right\|^{2},
\end{aligned}
$$

where we have used Theorem 3.1 , see $(24)$ in order to bound $\left\|A_{\theta}^{1 / 2} D_{N, \theta}^{1 / 2} \boldsymbol{w}\right\|^{2}$ with a suitable constant $C$.

We neglect in the left-hand side the term $\frac{\nu}{2}\left\|\nabla A_{\theta}^{1 / 2} D_{N, \theta}^{1 / 2} \boldsymbol{V}\right\|^{2}$ and we apply Gronwall's Lemma to obtain, for all $t \in[0, T)$,

$$
\begin{aligned}
\left\|A_{\theta}^{1 / 2} D_{N, \theta}^{1 / 2} \boldsymbol{V}(t)\right\|^{2} & \leq\left\|A_{\theta}^{1 / 2} D_{N, \theta}^{1 / 2} \boldsymbol{V}(0)\right\|^{2} \mathrm{e}^{\int_{0}^{t} \frac{C}{\nu}(N+1)^{3 a} d s} \\
& \leq\left\|A_{\theta}^{1 / 2} D_{N, \theta}^{1 / 2} \boldsymbol{V}(0)\right\|^{2} \mathrm{e}^{C T}
\end{aligned}
$$


Coming back to (51) and integrating in time over $(0, t)$ and using again (52), we get

$$
\begin{aligned}
\left\|A_{\theta}^{1 / 2} D_{N, \theta}^{1 / 2} \boldsymbol{V}(t)\right\|^{2}+\nu \int_{0}^{t}\left\|\nabla A_{\theta}^{1 / 2} D_{N, \theta}^{1 / 2} \boldsymbol{V}(s)\right\|^{2} \mathrm{~d} s \\
\leq\left\|A_{\theta}^{1 / 2} D_{N, \theta}^{1 / 2} \boldsymbol{V}(0)\right\|^{2}+\frac{C}{\nu}(N+1)^{3 a} \int_{0}^{t}\left\|A_{\theta}^{1 / 2} D_{N, \theta}^{1 / 2} \boldsymbol{V}(s)\right\|^{2} \mathrm{~d} s \\
\leq\left\|A_{\theta}^{1 / 2} D_{N, \theta}^{1 / 2} \boldsymbol{V}(0)\right\|^{2}+\frac{C}{\nu}(N+1)^{3 a} \mathrm{e}^{C T}\left\|A_{\theta}^{1 / 2} D_{N, \theta}^{1 / 2} \boldsymbol{V}(0)\right\|^{2} T \\
\quad=\tilde{C}\left\|A_{\theta}^{1 / 2} D_{N, \theta}^{1 / 2} \boldsymbol{V}(0)\right\|^{2}
\end{aligned}
$$

where $\tilde{C}=1+\frac{C T}{\nu}(N+1)^{3 a} \mathrm{e}^{C T}$.

We are going to prove the announced estimate (46).

Lemma 5.2. Assume $\theta \geq 1 / 2$. There exists a constant $\tilde{C}_{1}=\tilde{C}_{1}\left(T, N, \theta, K_{1}\right)>$ 0 such that, for each $t \in[0, T)$

$$
\left\|A_{\theta}^{1 / 2} D_{N, \theta}^{1 / 2} \boldsymbol{\eta}(t)\right\| \leq \tilde{C}_{1}\left\|A_{\theta}^{1 / 2} D_{N, \theta}^{1 / 2}\left(\boldsymbol{w}_{0}-\boldsymbol{v}_{0}\right)\right\|^{2} .
$$

Proof. The equations satisfied by $\boldsymbol{\eta}$ are

$$
\begin{aligned}
& \partial_{t} \boldsymbol{\eta}-\nu \Delta \boldsymbol{\eta}+\nabla Q_{1} \\
& \quad=\overline{\left(D_{N, \theta} \boldsymbol{V} \cdot \nabla\right) D_{N, \theta} \boldsymbol{V}-\left(D_{N, \theta} \boldsymbol{\eta} \cdot \nabla\right) D_{N, \theta} \boldsymbol{w}-\left(D_{N, \theta} \boldsymbol{w} \cdot \nabla\right) D_{N, \theta} \boldsymbol{\eta}} \\
& \nabla \cdot \boldsymbol{\eta}=0, \quad \boldsymbol{\eta}(0)=0,
\end{aligned}
$$

for a suitable $Q_{1}$ depending on $q, q_{1}$ and $Q$ in the Eq. (45).

Testing (53) by $A_{\theta} D_{N, \theta} \boldsymbol{\eta}$ and recalling that $\left(\left(D_{N, \theta} \boldsymbol{w} \cdot \nabla\right) D_{N, \theta} \boldsymbol{\eta}, D_{N, \theta} \boldsymbol{\eta}\right)$ $=0$ since $\nabla \cdot \boldsymbol{w}=0$, we obtain

$$
\begin{aligned}
& \frac{1}{2} \frac{\mathrm{d}}{\mathrm{d} t}\left\|A_{\theta}^{1 / 2} D_{N, \theta}^{1 / 2} \boldsymbol{\eta}\right\|^{2}+\nu\left\|\nabla A_{\theta}^{1 / 2} D_{N, \theta}^{1 / 2} \boldsymbol{\eta}\right\|^{2} \\
& \quad=\left(\left(D_{N, \theta} \boldsymbol{V} \cdot \nabla\right) D_{N, \theta} \boldsymbol{V}, D_{N, \theta} \boldsymbol{\eta}\right)-\left(\left(D_{N, \theta} \boldsymbol{\eta} \cdot \nabla\right) D_{N, \theta} \boldsymbol{w}, D_{N, \theta} \boldsymbol{\eta}\right) \\
& \quad=I+I I .
\end{aligned}
$$

Let us firstly estimate $I I$. Observing that from Lemma A.1

$$
I I=\left(\left(D_{N, \theta} \boldsymbol{\eta} \cdot \nabla\right) D_{N, \theta} \boldsymbol{\eta}, D_{N, \theta} \boldsymbol{w}\right),
$$

we apply the same arguments used to provide the estimate (50), to get

$$
|I I| \leq \frac{\nu}{2}\left\|\nabla A_{\theta}^{1 / 2} D_{N, \theta}^{1 / 2} \boldsymbol{\eta}\right\|^{2}+\frac{C}{\nu}(N+1)^{3 a}\left\|A_{\theta}^{1 / 2} D_{N, \theta}^{1 / 2} \boldsymbol{w}\right\|^{2}\left\|A_{\theta}^{1 / 2} D_{N, \theta}^{1 / 2} \boldsymbol{\eta}\right\|^{2},
$$

where $a$ is the same value involved by the Gagliardo-Nirenberg inequality (95). 
As regards to $I$, we use once again Hölder's inequality, the estimates (95), (17), (18) and Young's inequality to get

$$
\begin{aligned}
|I| \leq & \left\|D_{N, \theta} \boldsymbol{V}\right\|_{\mathrm{L}^{3}}\left\|\nabla D_{N, \theta} \boldsymbol{V}\right\|_{\mathrm{L}^{3}}\left\|D_{N, \theta} \boldsymbol{\eta}\right\|_{\mathrm{L}^{3}} \\
\leq & \left\|D_{N, \theta} \boldsymbol{V}\right\|^{1-a}\left\|A_{\theta}^{1 / 2} D_{N, \theta} \boldsymbol{V}\right\|^{a}\left\|\nabla D_{N, \theta} \boldsymbol{V}\right\|^{1-a}\left\|\nabla A_{\theta}^{1 / 2} D_{N, \theta} \boldsymbol{V}\right\|^{a} \\
& \times\left\|D_{N, \theta} \boldsymbol{\eta}\right\|^{1-a}\left\|A_{\theta}^{1 / 2} D_{N, \theta} \boldsymbol{\eta}\right\|^{a} \\
\leq & C(N+1)^{\frac{3}{2} a}\left\|A_{\theta}^{1 / 2} D_{N, \theta}^{1 / 2} \boldsymbol{V}\right\|\left\|\nabla A_{\theta}^{1 / 2} D_{N, \theta}^{1 / 2} \boldsymbol{V}\right\|\left\|A_{\theta}^{1 / 2} D_{N, \theta}^{1 / 2} \boldsymbol{\eta}\right\| \\
\leq & C \nu\left\|A_{\theta}^{1 / 2} D_{N, \theta}^{1 / 2} \boldsymbol{V}\right\|^{2}\left\|\nabla A_{\theta}^{1 / 2} D_{N, \theta}^{1 / 2} \boldsymbol{V}\right\|^{2}+\frac{C}{\nu}(N+1)^{3 a}\left\|A_{\theta}^{1 / 2} D_{N, \theta}^{1 / 2} \boldsymbol{\eta}\right\|^{2} .
\end{aligned}
$$

Coming back to (54), using (55) and (56), we get

$$
\begin{aligned}
\frac{1}{2} \frac{\mathrm{d}}{\mathrm{d} t} & \left\|A_{\theta}^{1 / 2} D_{N, \theta}^{1 / 2} \boldsymbol{\eta}\right\|^{2}+\nu\left\|\nabla A_{\theta}^{1 / 2} D_{N, \theta}^{1 / 2} \boldsymbol{\eta}\right\|^{2} \\
\leq & \frac{\nu}{2}\left\|\nabla A_{\theta}^{1 / 2} D_{N, \theta}^{1 / 2} \boldsymbol{\eta}\right\|^{2}+\frac{C}{\nu}(N+1)^{3 a}\left\|A_{\theta}^{1 / 2} D_{N, \theta}^{1 / 2} \boldsymbol{w}\right\|^{2}\left\|A_{\theta}^{1 / 2} D_{N, \theta}^{1 / 2} \boldsymbol{\eta}\right\|^{2} \\
& +C \nu\left\|A_{\theta}^{1 / 2} D_{N, \theta}^{1 / 2} \boldsymbol{V}\right\|^{2}\left\|\nabla A_{\theta}^{1 / 2} D_{N, \theta}^{1 / 2} \boldsymbol{V}\right\|^{2}+\frac{C}{\nu}(N+1)^{3 a}\left\|A_{\theta}^{1 / 2} D_{N, \theta}^{1 / 2} \boldsymbol{\eta}\right\|^{2} \\
= & \frac{\nu}{2}\left\|\nabla A_{\theta}^{1 / 2} D_{N, \theta}^{1 / 2} \boldsymbol{\eta}\right\|^{2}+\frac{C}{\nu}(N+1)^{3 a}\left(\left\|A_{\theta}^{1 / 2} D_{N, \theta}^{1 / 2} \boldsymbol{w}\right\|^{2}+1\right)\left\|A_{\theta}^{1 / 2} D_{N, \theta}^{1 / 2} \boldsymbol{\eta}\right\|^{2} \\
& +C \nu\left\|A_{\theta}^{1 / 2} D_{N, \theta}^{1 / 2} \boldsymbol{V}\right\|^{2}\left\|\nabla A_{\theta}^{1 / 2} D_{N, \theta}^{1 / 2} \boldsymbol{V}\right\|^{2} \\
\leq & \frac{\nu}{2}\left\|\nabla A_{\theta}^{1 / 2} D_{N, \theta}^{1 / 2} \boldsymbol{\eta}\right\|^{2}+\frac{C}{\nu}(N+1)^{3 a}\left\|A_{\theta}^{1 / 2} D_{N, \theta}^{1 / 2} \boldsymbol{\eta}\right\|^{2} \\
& +C \tilde{C} \nu\left\|A_{\theta}^{1 / 2} D_{N, \theta}^{1 / 2} \boldsymbol{V}(0)\right\|^{2}\left\|\nabla A_{\theta}^{1 / 2} D_{N, \theta}^{1 / 2} \boldsymbol{V}\right\|^{2},
\end{aligned}
$$

where we have used Theorem 3.1 ; see $(24)$ in order to bound $\left\|A_{\theta}^{1 / 2} D_{N, \theta}^{1 / 2} \boldsymbol{w}\right\|^{2}$ with a suitable constant $C$ and Lemma 5.1 to bound $\left\|A_{\theta}^{1 / 2} D_{N, \theta}^{1 / 2} \boldsymbol{V}\right\|^{2}$ with $\tilde{C}\left\|A_{\theta}^{1 / 2} D_{N, \theta}^{1 / 2} \boldsymbol{V}(0)\right\|^{2}$.

Absorbing $\frac{\nu}{2}\left\|\nabla A_{\theta}^{1 / 2} D_{N, \theta}^{1 / 2} \boldsymbol{\eta}\right\|^{2}$ in the left-hand side of (57) we get

$$
\begin{aligned}
& \frac{1}{2} \frac{\mathrm{d}}{\mathrm{d} t}\left\|A_{\theta}^{1 / 2} D_{N, \theta}^{1 / 2} \boldsymbol{\eta}\right\|^{2}+\frac{\nu}{2}\left\|\nabla A_{\theta}^{1 / 2} D_{N, \theta}^{1 / 2} \boldsymbol{\eta}\right\|^{2} \\
& \quad \leq \frac{C}{\nu}(N+1)^{3 a}\left\|A_{\theta}^{1 / 2} D_{N, \theta}^{1 / 2} \boldsymbol{\eta}\right\|^{2}+\tilde{C} \nu\left\|A_{\theta}^{1 / 2} D_{N, \theta}^{1 / 2} \boldsymbol{V}(0)\right\|^{2}\left\|\nabla A_{\theta}^{1 / 2} D_{N, \theta}^{1 / 2} \boldsymbol{V}\right\|^{2} .
\end{aligned}
$$

Neglecting $\frac{\nu}{2}\left\|\nabla A_{\theta}^{1 / 2} D_{N, \theta}^{1 / 2} \boldsymbol{\eta}\right\|^{2}$ in the left-hand side of (58) and integrating in time over $(0, t)$ (recall that $\boldsymbol{\eta}(0)=\mathbf{0}$ ) we get

$$
\begin{gathered}
\left\|A_{\theta}^{1 / 2} D_{N, \theta}^{1 / 2} \boldsymbol{\eta}(t)\right\|^{2} \leq \frac{C}{\nu}(N+1)^{3 a} \int_{0}^{t}\left\|A_{\theta}^{1 / 2} D_{N, \theta}^{1 / 2} \boldsymbol{\eta}(s)\right\|^{2} \mathrm{~d} s \\
+\tilde{C}\left\|A_{\theta}^{1 / 2} D_{N, \theta}^{1 / 2} \boldsymbol{V}(0)\right\|^{2} \nu \int_{0}^{t}\left\|\nabla A_{\theta}^{1 / 2} D_{N, \theta}^{1 / 2} \boldsymbol{V}(s)\right\|^{2} \mathrm{~d} s \\
\leq C_{N} \int_{0}^{t}\left\|A_{\theta}^{1 / 2} D_{N, \theta}^{1 / 2} \boldsymbol{\eta}(s)\right\|^{2} d s+\tilde{C}^{2}\left\|A_{\theta}^{1 / 2} D_{N, \theta}^{1 / 2} \boldsymbol{V}(0)\right\|^{4},
\end{gathered}
$$

with $C_{N}:=\frac{C}{\nu}(N+1)^{3 a}$ and in view of Lemma 5.1. 

$[0, T)$

Applying then Gronwall's lemma to the last inequality we get for $t \in$

$$
\left\|A_{\theta}^{1 / 2} D_{N, \theta}^{1 / 2} \boldsymbol{\eta}(t)\right\|^{2} \leq \tilde{C}^{2}\left\|A_{\theta}^{1 / 2} D_{N, \theta}^{1 / 2} \boldsymbol{V}(0)\right\|^{4} \mathrm{e}^{C_{N} t} \leq \tilde{C}_{1}\left\|A_{\theta}^{1 / 2} D_{N, \theta}^{1 / 2} \boldsymbol{V}(0)\right\|^{4},
$$

where $\tilde{C}_{1}:=\tilde{C}^{2} \mathrm{e}^{C_{N} T}$. This ends the proof.

\section{Estimate for the global attractor dimension}

First, we linearize Eq. (3), projected onto the space of divergence free functions, about a solution $\boldsymbol{w}$. The linear equation satisfied by a perturbation $\delta \boldsymbol{w}$ is

$$
\partial_{t} \delta \boldsymbol{w}+\overline{\nabla \cdot\left(D_{N, \theta} \boldsymbol{w} \otimes D_{N, \theta} \delta \boldsymbol{w}\right)}+\overline{\nabla \cdot\left(D_{N, \theta} \delta \boldsymbol{w} \otimes D_{N, \theta} \boldsymbol{w}\right)}-\nu \Delta \delta \boldsymbol{w}=\mathbf{0},
$$

that is to say

$$
\frac{\mathrm{d}}{\mathrm{d} t} \delta \boldsymbol{w}+T(t) \delta \boldsymbol{w}=\mathbf{0},
$$

where

$$
T(t)=-\nu \Delta+\overline{\nabla \cdot\left(D_{N, \theta} \boldsymbol{w} \otimes(\cdot)\right)}+\overline{\nabla \cdot\left((\cdot) \otimes D_{N, \theta} \boldsymbol{w}\right)} .
$$

If $\delta \boldsymbol{w}_{j, 0}, j=1, \ldots, M$, are $M$ linearly independent functions in $\mathbf{H}_{\sigma}^{\theta}$, we denote by $\delta \boldsymbol{w}_{j}(t)$ the corresponding solutions to (59), computed at time $t$, with $\delta \boldsymbol{w}_{j}(0)=\delta \boldsymbol{w}_{j, 0}$. We set $E=\left\{\delta \boldsymbol{w}_{1}(t), \ldots, \delta \boldsymbol{w}_{M}(t)\right\}$ and denote by $P_{M}(t)$ the orthogonal projection of $\mathbf{H}_{\sigma}^{\theta}$ onto the span of $E$, denoted hereafter by $\langle E\rangle$.

In this section we provide $\mathbf{H}_{\sigma}^{\theta}$ with the inner product

$$
[\boldsymbol{u}, \boldsymbol{v}]:=\left(A_{\theta}^{1 / 2} D_{N, \theta}^{1 / 2} \boldsymbol{u}, A_{\theta}^{1 / 2} D_{N, \theta}^{1 / 2} \boldsymbol{v}\right)
$$

and introduce the operator $\mathcal{T}_{M}(t)$ defined by

$$
\mathcal{T}_{M}(t)=\operatorname{Trace}\left(P_{M}(t) \circ T(t) \circ P_{M}(t)\right) .
$$

Let $\left\{\boldsymbol{\varphi}_{j}(t)\right\}_{j=1}^{M}$ be an orthonormal (with respect to $[\cdot, \cdot]$ defined in (61)) basis of $\langle E\rangle=P_{M}(t) \mathbf{H}_{\sigma}^{\theta}$, so that

$$
\left[\boldsymbol{\varphi}_{i}(t), \boldsymbol{\varphi}_{j}(t)\right]=\delta_{i j}(\text { Kronecker symbol }), \quad i, j=1, \ldots, M .
$$

Using (62) and (60) we get

$$
\begin{aligned}
\mathcal{T}_{M}(t)= & \sum_{i=1}^{M}\left[T(t) \boldsymbol{\varphi}_{i}(t), \boldsymbol{\varphi}_{i}(t)\right] \\
= & \sum_{i=1}^{M}\left[-\nu \Delta \boldsymbol{\varphi}_{i}(t)+\overline{\nabla \cdot\left(D_{N, \theta} \boldsymbol{w} \otimes D_{N, \theta} \boldsymbol{\varphi}_{i}(t)\right)}\right. \\
& \left.+\overline{\nabla \cdot\left(D_{N, \theta} \boldsymbol{\varphi}_{i}(t) \otimes D_{N, \theta} \boldsymbol{w}\right)}, \boldsymbol{\varphi}_{i}(t)\right]
\end{aligned}
$$


According to (61) we have that

$$
\begin{aligned}
& {\left[-\nu \Delta \boldsymbol{\varphi}_{i}(t), \boldsymbol{\varphi}_{i}(t)\right]=\left(-\nu \Delta A_{\theta}^{1 / 2} D_{N, \theta}^{1 / 2} \boldsymbol{\varphi}_{i}(t), A_{\theta}^{1 / 2} D_{N, \theta}^{1 / 2} \boldsymbol{\varphi}_{i}(t)\right)} \\
& =\left(\nu \nabla A_{\theta}^{1 / 2} D_{N, \theta}^{1 / 2} \boldsymbol{\varphi}_{i}(t), \nabla A_{\theta}^{1 / 2} D_{N, \theta}^{1 / 2} \boldsymbol{\varphi}_{i}(t)\right)=\nu\left\|\nabla A_{\theta}^{1 / 2} D_{N, \theta}^{1 / 2} \boldsymbol{\varphi}_{i}(t)\right\|^{2}, \\
& {\left[\overline{\nabla \cdot\left(D_{N, \theta} \boldsymbol{w} \otimes D_{N, \theta} \boldsymbol{\varphi}_{i}(t)\right)}, \boldsymbol{\varphi}_{i}(t)\right]} \\
& =\left(A_{\theta}^{1 / 2} D_{N, \theta}^{1 / 2} \overline{\nabla \cdot\left(D_{N, \theta} \boldsymbol{w} \otimes D_{N, \theta} \boldsymbol{\varphi}_{i}(t)\right)}, A_{\theta}^{1 / 2} D_{N, \theta}^{1 / 2} \boldsymbol{\varphi}_{i}(t)\right) \\
& =\left(A_{\theta} \overline{\nabla \cdot\left(D_{N, \theta} \boldsymbol{w} \otimes D_{N, \theta} \boldsymbol{\varphi}_{i}(t)\right)}, D_{N, \theta} \boldsymbol{\varphi}_{i}(t)\right) \\
& =\left(\nabla \cdot\left(D_{N, \theta} \boldsymbol{w} \otimes D_{N, \theta} \boldsymbol{\varphi}_{i}(t)\right), D_{N, \theta} \boldsymbol{\varphi}_{i}(t)\right) \\
& =\left(\left(D_{N, \theta} \boldsymbol{\varphi}_{i}(t) \cdot \nabla\right) D_{N, \theta} \boldsymbol{w}, D_{N, \theta} \boldsymbol{\varphi}_{i}(t)\right), \\
& {\left[\overline{\nabla \cdot\left(D_{N, \theta} \boldsymbol{\varphi}_{i}(t) \otimes D_{N, \theta} \boldsymbol{w}\right)}, \boldsymbol{\varphi}_{i}(t)\right]} \\
& =\left(A_{\theta}^{1 / 2} D_{N, \theta}^{1 / 2} \overline{\nabla \cdot\left(D_{N, \theta} \boldsymbol{\varphi}_{i}(t) \otimes D_{N, \theta} \boldsymbol{w}\right)}, A_{\theta}^{1 / 2} D_{N, \theta}^{1 / 2} \boldsymbol{\varphi}_{i}(t)\right) \\
& =\left(A_{\theta} \overline{\nabla \cdot\left(D_{N, \theta} \boldsymbol{\varphi}_{i}(t) \otimes D_{N, \theta} \boldsymbol{w}\right)}, D_{N, \theta} \boldsymbol{\varphi}_{i}(t)\right) \\
& =\left(\nabla \cdot\left(D_{N, \theta} \boldsymbol{\varphi}_{i}(t) \otimes D_{N, \theta} \boldsymbol{w}\right), D_{N, \theta} \boldsymbol{\varphi}_{i}(t)\right)=0,
\end{aligned}
$$

where integration by parts and formulas (91), (93) have been used in the calculations above (remember that $\nabla \cdot \boldsymbol{w}=\nabla \cdot \boldsymbol{\varphi}_{i}=0$ ).

Substituting (64)-(66) into (63) we get

$$
\begin{aligned}
\mathcal{T}_{M}(t) & =\sum_{i=1}^{M} \nu\left\|\nabla A_{\theta}^{1 / 2} D_{N, \theta}^{1 / 2} \boldsymbol{\varphi}_{i}(t)\right\|^{2}+\sum_{i=1}^{M}\left(\left(D_{N, \theta} \boldsymbol{\varphi}_{i}(t) \cdot \nabla\right) D_{N, \theta} \boldsymbol{w}, D_{N, \theta} \boldsymbol{\varphi}_{i}(t)\right) \\
& =Q_{M}(t)+\mathcal{R}_{M}(t) .
\end{aligned}
$$

The next goal is to prove that

$$
X_{M}:=\liminf _{T \rightarrow+\infty} \frac{1}{T} \int_{0}^{T} \mathcal{T}_{M}(t) d t>0,
$$

provided that $M$ is taken sufficiently large, and find an estimate for $M$ (see the next Lemma 6.5). In fact (see [19, Sect. 3.4] and [10]) if $M$ is sufficiently large such that (68) is satisfied, then $M$ is an upper bound for the Hausdorff dimension $d_{\mathrm{H}}(\mathcal{A})$ and the fractal dimension $d_{\mathrm{F}}(\mathcal{A})$ of the global attractor $\mathcal{A}$ :

$$
d_{\mathrm{H}}(\mathcal{A}) \leq d_{\mathrm{F}}(\mathcal{A}) \leq M
$$

To get this result, we need to provide useful estimates for $Q_{M}(t)$ and $\mathcal{R}_{M}(t)$ in the representation $(67)$ of $\mathcal{T}_{M}(t)$.

Lemma 6.1. Assume that $1 / 2 \leq \theta \leq 1$. There exists a numerical constant $C>0$ such that 


$$
Q_{M}(t) \geq \frac{\nu \lambda_{1} M^{5 / 3}}{C},
$$

where $\lambda_{1}=L^{-2}$ is the first eigenvalue of $-\Delta$.

Proof. We use the Lieb-Thirring inequality in Lemma A.5 for

$$
\boldsymbol{\theta}_{j}(\boldsymbol{x})=A_{\theta}^{1 / 2} D_{N, \theta}^{1 / 2} \boldsymbol{\varphi}_{j}(t, \boldsymbol{x}) .
$$

By (62) it follows that $\left\{\boldsymbol{\theta}_{j}\right\}_{j=1}^{M}$ defined above is an orthonormal set in $\mathrm{L}_{\sigma}^{2}\left(\mathbb{T}^{3}\right)$. For the functions $\boldsymbol{\theta}_{j}$ above, the right-hand side of Lieb-Thirring's inequality (105) becomes

$$
\begin{aligned}
& \sum_{j=1}^{M} \int_{\mathbb{T}^{3}} \nabla A_{\theta}^{1 / 2} D_{N, \theta}^{1 / 2} \boldsymbol{\varphi}_{j}(t, \boldsymbol{x}): \nabla A_{\theta}^{1 / 2} D_{N, \theta}^{1 / 2} \boldsymbol{\varphi}_{j}(t, \boldsymbol{x}) \mathrm{d} \boldsymbol{x} \\
& \quad=\sum_{j=1}^{M} \int_{\mathbb{T}^{3}}\left|\nabla A_{\theta}^{1 / 2} D_{N, \theta}^{1 / 2} \boldsymbol{\varphi}_{j}(t, \boldsymbol{x})\right|^{2} \mathrm{~d} \boldsymbol{x}=\sum_{j=1}^{M}\left\|\nabla A_{\theta}^{1 / 2} D_{N, \theta}^{1 / 2} \boldsymbol{\varphi}_{j}(t)\right\|^{2} \\
& \quad=\frac{Q_{M}(t)}{\nu}
\end{aligned}
$$

As regards to the left-hand side of (105) we get by the use of Jensen's inequality

$$
\begin{aligned}
& \int_{\mathbb{T}^{3}}\left(\sum_{j=1}^{M}\left|A_{\theta}^{1 / 2} D_{N, \theta}^{1 / 2} \boldsymbol{\varphi}_{j}(t, \boldsymbol{x})\right|^{2}\right)^{5 / 3} \mathrm{~d} \boldsymbol{x} \\
& \geq \frac{1}{\left|\mathbb{T}^{3}\right|^{2 / 3}}\left(\int_{\mathbb{T}^{3}} \sum_{j=1}^{M}\left|A_{\theta}^{1 / 2} D_{N, \theta}^{1 / 2} \boldsymbol{\varphi}_{j}(t, \boldsymbol{x})\right|^{2} \mathrm{~d} \boldsymbol{x}\right)^{5 / 3} \\
& =\frac{1}{\left|\mathbb{T}^{3}\right|^{2 / 3}}\left(\sum_{j=1}^{M}\left\|A_{\theta}^{1 / 2} D_{N, \theta}^{1 / 2} \boldsymbol{\varphi}_{j}(t)\right\|^{2}\right)^{5 / 3}=\frac{1}{\left|\mathbb{T}^{3}\right|^{2 / 3}} M^{5 / 3}
\end{aligned}
$$

where $\left|\mathbb{T}^{3}\right|=(2 \pi L)^{3}$ is the Lebesgue measure of the torus $\mathbb{T}^{3}$, and where we used that $\left\|A_{\theta}^{1 / 2} D_{N, \theta}^{1 / 2} \varphi_{j}(t)\right\|=1$ (because of the orthonormality).

The estimate (69) comes from combining Lieb-Thirring's inequality with the estimate in (70).

Remark 6.2. We notice that the same estimate (69) could be obtained by the following alternative argument. The asymptotic behavior of the eigenvalues of the operator $-\Delta$ is such that (see $[10,19]$ )

$$
\lambda_{i} \geq \frac{\lambda_{1} i^{2 / 3}}{C}, \quad i=1,2, \ldots \quad \text { and } \quad Q_{M}(t) \geq \nu \sum_{i=1}^{M} \lambda_{i} .
$$

Hence $Q_{M}(t) \geq \frac{\nu \lambda_{1} M^{5 / 3}}{C}$ since, by induction, one can easily prove that $\frac{5}{3} \sum_{i=1}^{M} i^{2 / 3} \geq M^{5 / 3}$ 
Lemma 6.3. Assume that $1 / 2<\theta \leq 1$ and let $\sigma=\sigma(\theta)$ be defined as in (94). Then the following estimate holds

$$
\left|\mathcal{R}_{M}(t)\right| \leq \frac{Q_{M}(t)}{2}+C C_{1} M^{(1-\sigma) s}\left\|\nabla A_{\theta}^{1 / 2} D_{N, \theta}^{1 / 2} \boldsymbol{w}\right\|^{2}\left\|A_{\theta}^{1 / 2} D_{N, \theta}^{1 / 2} \boldsymbol{w}\right\|^{2 \mu}
$$

where

$$
C_{1}:=\left(\frac{(N+1)^{\sigma+1 / 2} L^{3 / q}}{\alpha^{2 \sigma \theta} \nu^{\sigma(3 / 2-\theta)}}\right)^{s},
$$

for a suitable numerical constant $C>0$, and

$$
\mu:=\frac{-1+3 \sigma-2 \sigma \theta}{2-3 \sigma+2 \sigma \theta}, \quad q:=\frac{6}{2(1+2 \sigma) \theta-1}, \quad s:=\frac{2}{2-3 \sigma+2 \sigma \theta} .
$$

Proof. For a given $\theta$, such that $1 / 2<\theta \leq 1$, let $\sigma=\sigma(\theta)$ be fixed as in the statement above. The Cauchy-Schwarz and the Hölder inequalities together with (17) give

$$
\begin{aligned}
& \left|\mathcal{R}_{M}(t)\right| \leq \int_{\mathbb{T}^{3}}\left|\sum_{i=1}^{M}\left(D_{N, \theta} \boldsymbol{\varphi}_{i}(t) \cdot \nabla\right) D_{N, \theta} \boldsymbol{w} \cdot D_{N, \theta} \boldsymbol{\varphi}_{i}(t)\right| \mathrm{d} \boldsymbol{x} \\
& \leq \int_{\mathbb{T}^{3}} \sum_{i=1}^{M}\left|D_{N, \theta} \boldsymbol{\varphi}_{i}(t)\right|^{2}\left|\nabla D_{N, \theta} \boldsymbol{w}\right| \mathrm{d} \boldsymbol{x} \\
& \leq \int_{\mathbb{T}^{3}}\left(\sum_{i=1}^{M}\left|D_{N, \theta} \boldsymbol{\varphi}_{i}(t)\right|^{2}\right)^{\sigma}\left(\sum_{i=1}^{M}\left|D_{N, \theta} \boldsymbol{\varphi}_{i}(t)\right|^{2}\right)^{1-\sigma}\left|\nabla D_{N, \theta} \boldsymbol{w}\right| \mathrm{d} \boldsymbol{x} \\
& \leq\left\|\left(\sum_{i=1}^{M}\left|D_{N, \theta} \boldsymbol{\varphi}_{i}(t)\right|^{2}\right)^{\sigma}\right\|_{L^{\infty}}\left\|\left(\sum_{i=1}^{M}\left|D_{N, \theta} \boldsymbol{\varphi}_{i}(t)\right|^{2}\right)^{1-\sigma}\right\|_{L^{\frac{1}{1-\sigma}}} \\
& \times\left\|\nabla D_{N, \theta} \boldsymbol{w}\right\|_{L^{p}}\left|\mathbb{T}^{3}\right|^{1 / q} \\
& \leq C\left\|\sum_{i=1}^{M}\left|D_{N, \theta} \boldsymbol{\varphi}_{i}(t)\right|^{2}\right\|_{L^{\infty}}^{\sigma}\left(\sum_{i=1}^{M}\left\|D_{N, \theta} \boldsymbol{\varphi}_{i}(t)\right\|^{2}\right)^{1-\sigma}\left\|\nabla D_{N, \theta} \boldsymbol{w}\right\|_{L^{p}} L^{3 / q} \\
& \leq C\left\|\sum_{i=1}^{M}\left|D_{N, \theta} \boldsymbol{\varphi}_{i}(t)\right|^{2}\right\|_{L^{\infty}}^{\sigma}\left(\sum_{i=1}^{M}\left\|A_{\theta}^{1 / 2} D_{N, \theta}^{1 / 2} \boldsymbol{\varphi}_{i}(t)\right\|^{2}\right)^{1-\sigma} \\
& \times\left\|\nabla D_{N, \theta} \boldsymbol{w}\right\|_{L^{p}} L^{3 / q} \\
& =C\left\|\sum_{i=1}^{M}\left|D_{N, \theta} \boldsymbol{\varphi}_{i}(t)\right|^{2}\right\|_{L^{\infty}}^{\sigma} M^{1-\sigma}\left\|\nabla D_{N, \theta} \boldsymbol{w}\right\|_{L^{p}} L^{3 / q},
\end{aligned}
$$

where $p$ is the exponent defined by (100), through $\sigma$ and $\theta$, while $q$ is given in (73) and makes satisfied the needed identity

$$
\frac{1}{p}+\frac{1}{q}=\sigma
$$


It is understood that the splitting of $\sum_{i=1}^{M}\left|D_{N, \theta} \boldsymbol{\varphi}_{i}(t)\right|^{2}$ into the $\sigma$ and $(1-\sigma)$ powers becomes trivial as long as $\sigma=1$ [when $2 / 3 \leq \theta \leq 1$, cf. (94)]. In this case $L^{\infty}$ and $L^{\frac{1}{1-\sigma}}$-norms of those two powers "collapse" into the $L^{\infty}$-norm of the whole $\sum_{i=1}^{M}\left|D_{N, \theta} \varphi_{i}(t)\right|^{2}$ (thus $M^{1-\sigma}$ in the last expression above reduces to 1), while $p$ and $q$ become the conjugate exponents

$$
p=\frac{6}{7-6 \theta}, \quad q=\frac{6}{6 \theta-1} .
$$

Now, we use Lemma A.4 in the Appendix to estimate the $L^{\infty}$-norm in the right-hand side of (74) by

$$
\left\|\sum_{i=1}^{M}\left|D_{N, \theta} \boldsymbol{\varphi}_{i}(t)\right|^{2}\right\|_{L^{\infty}} \leq \frac{C}{\alpha^{2 \theta}}(N+1)\left(\frac{Q_{M}(t)}{\nu}\right)^{3 / 2-\theta},
$$

for a suitable numerical constant $C$. It is just the application of the above inequality (see Lemma A.3) that requires to exclude the border value $\theta=1 / 2$ from our subsequent considerations.

By the use of (98) in Lemma A.2 in the Appendix, we estimate the $L^{p}$ norm of $\nabla D_{N, \theta} \boldsymbol{w}$ as

$$
\left\|\nabla D_{N, \theta} \boldsymbol{w}\right\|_{L^{p}} \leq C\left\|\nabla A_{\theta}^{1 / 2} D_{N, \theta} \boldsymbol{w}\right\|^{\beta}\left\|A_{\theta}^{1 / 2} D_{N, \theta} \boldsymbol{w}\right\|^{1-\beta},
$$

with $\beta$ given in (100). Then we apply estimates (18), (19) to get

$$
\left\|\nabla D_{N, \theta} \boldsymbol{w}\right\|_{L^{p}} \leq C(N+1)^{1 / 2}\left\|\nabla A_{\theta}^{1 / 2} D_{N, \theta}^{1 / 2} \boldsymbol{w}\right\|^{\beta}\left\|A_{\theta}^{1 / 2} D_{N, \theta}^{1 / 2} \boldsymbol{w}\right\|^{1-\beta} .
$$

From (74), (75) and (76) we find

$$
\begin{aligned}
& \left|R_{M}(t)\right| \\
& \qquad \leq \frac{C(N+1)^{\sigma+1 / 2} L^{3 / q} M^{1-\sigma}}{\alpha^{2 \sigma \theta}}\left(\frac{Q_{M}(t)}{\nu}\right)^{\sigma(3 / 2-\theta)}\left\|\nabla A_{\theta}^{1 / 2} D_{N, \theta}^{1 / 2} \boldsymbol{w}\right\|^{\beta} \\
& \quad \times\left\|A_{\theta}^{1 / 2} D_{N, \theta}^{1 / 2} \boldsymbol{w}\right\|^{1-\beta} .
\end{aligned}
$$

Now, we apply to the last expression above the Young inequality $a b \leq \frac{a^{r}}{r}+\frac{b^{s}}{s}$, where the conjugate exponents $r=\frac{1}{\sigma(3 / 2-\theta)}=\frac{2}{\sigma(3-2 \theta)}$ and $s=\frac{1}{1-\sigma(3 / 2-\theta)}=$ $\frac{2}{2-3 \sigma+2 \sigma \theta}$ are chosen in such a way that $\frac{a^{r}}{r}=\frac{Q_{M}(t)}{2}$; then we get

$$
\begin{aligned}
\left|\mathcal{R}_{M}(t)\right| \leq & \frac{Q_{M}(t)}{2}+C\left(\frac{(N+1)^{\sigma+1 / 2} L^{3 / q} M^{1-\sigma}}{\alpha^{2 \sigma \theta} \nu^{\sigma(3 / 2-\theta)}}\right)^{s}\left\|\nabla A_{\theta}^{1 / 2} D_{N, \theta}^{1 / 2} \boldsymbol{w}\right\|^{s \beta} \\
& \times\left\|A_{\theta}^{1 / 2} D_{N, \theta}^{1 / 2} \boldsymbol{w}\right\|^{s(1-\beta)}
\end{aligned}
$$

which gives the desired estimate in view of (72) and since $s \beta=2, s(1-\beta)=$ $2 \mu$. 
Remark 6.4. According to (94) one explicitly derives the following values of the exponent $\mu$ involved in the estimate (71) [see (73)]

$$
\mu= \begin{cases}\frac{\theta}{1-\theta}, & \text { if } 1 / 2 \leq \theta<2 / 3 \\ \frac{2(1-\theta)}{2 \theta-1}, & \text { if } 2 / 3 \leq \theta \leq 1\end{cases}
$$

In particular it follows that

$$
1 \leq \mu<2 \quad \text { as } 1 / 2 \leq \theta<2 / 3 \quad \text { and } \quad 0 \leq \mu \leq 2 \quad \text { as } 2 / 3 \leq \theta \leq 1 .
$$

In view of the subsequent analysis, it is also worth noticing that because of (94) and (73) one has

$0<(1-\sigma) s \leq 1 \quad$ as $1 / 2 \leq \theta<2 / 3 \quad$ and $\quad(1-\sigma) s=0 \quad$ as $2 / 3 \leq \theta \leq 1$.

Let us prove now that (68) holds. To this end, we are going to prove that the following lemma holds true.

Lemma 6.5. Assume that $1 / 2<\theta \leq 1$. Then

$$
X_{M}=\liminf _{T \rightarrow+\infty} \frac{1}{T} \int_{0}^{T} \mathcal{T}_{M}(t) d t \geq \frac{\nu \lambda_{1} M^{5 / 3}}{C}-M^{(1-\sigma) s} C_{1} C \frac{K_{1}^{1+\mu}}{\nu^{1+\mu} \lambda_{1}^{\mu}},
$$

where $C_{1}$ is defined in (72).

Proof. Let us start by the representation formula (67). In view of (71), we have the following estimate

$$
\begin{aligned}
\mathcal{T}_{M}(t)= & Q_{M}(t)+\mathcal{R}_{M}(t) \geq Q_{M}(t)-\left|\mathcal{R}_{M}(t)\right| \\
& \geq \frac{Q_{M}(t)}{2}-C C_{1} M^{(1-\sigma) s}\left\|\nabla A_{\theta}^{1 / 2} D_{N, \theta}^{1 / 2} \boldsymbol{w}\right\|^{2}\left\|A_{\theta}^{1 / 2} D_{N, \theta}^{1 / 2} \boldsymbol{w}\right\|^{2 \mu}
\end{aligned}
$$

where the exponents $\sigma, \mu$ and $s$ are given in (94) and (73). We recall that the use of estimate $(71)$ requires $\theta>1 / 2$. We calculate

$$
\begin{aligned}
X_{M}= & \liminf _{T \rightarrow+\infty} \frac{1}{T} \int_{0}^{T} \mathcal{T}_{M}(t) d t \\
\geq & \liminf _{T \rightarrow+\infty} \frac{1}{T} \int_{0}^{T} \frac{Q_{M}(t)}{2} d t \\
& -C C_{1} M^{(1-\sigma) s} \limsup _{T \rightarrow+\infty} \frac{1}{T} \int_{0}^{T}\left\|\nabla A_{\theta}^{1 / 2} D_{N, \theta}^{1 / 2} \boldsymbol{w}\right\|^{2}\left\|A_{\theta}^{1 / 2} D_{N, \theta}^{1 / 2} \boldsymbol{w}\right\|^{2 \mu} d t \\
\geq & \frac{\nu \lambda_{1} M^{5 / 3}}{C}-C C_{1} M^{(1-\sigma) s} \limsup _{T \rightarrow+\infty} \frac{1}{T} \int_{0}^{T}\left\|\nabla A_{\theta}^{1 / 2} D_{N, \theta}^{1 / 2} \boldsymbol{w}\right\|^{2} \\
& \times\left\|A_{\theta}^{1 / 2} D_{N, \theta}^{1 / 2} \boldsymbol{w}\right\|^{2 \mu} d t
\end{aligned}
$$

in view of (69). It remains to produce an estimate for the limit superior in the previous inequality. We will show (see the next Lemma 6.7) that the following estimate

$$
\limsup _{T \rightarrow+\infty} \frac{1}{T} \int_{0}^{T}\left\|\nabla A_{\theta}^{1 / 2} D_{N, \theta}^{1 / 2} \boldsymbol{w}\right\|^{2}\left\|A_{\theta}^{1 / 2} D_{N, \theta}^{1 / 2} \boldsymbol{w}\right\|^{2 \mu} d t \leq C \frac{K_{1}^{1+\mu}}{\nu^{1+\mu} \lambda_{1}^{\mu}}
$$


holds true. We use now the estimate above in (79) to find

$$
\begin{aligned}
X_{M} & =\liminf _{T \rightarrow+\infty} \frac{1}{T} \int_{0}^{T} \mathcal{T}_{M}(t) d t \\
& \geq \frac{\nu \lambda_{1} M^{5 / 3}}{C}-M^{(1-\sigma) s} C_{1} C \frac{K_{1}^{1+\mu}}{\nu^{1+\mu} \lambda_{1}^{\mu}},
\end{aligned}
$$

which is just the estimate (78) of the lemma.

Remark 6.6. The estimate (78) implies $X_{M}$ to be positive for $M>0$ large enough, since the exponent $(1-\sigma) s$ of the power of $M$ in the second term of the right-hand side above lies in the interval $(0,1]$ as long as $1 / 2<\theta \leq 1$, see Remark 6.4.

Lemma 6.7. Assume that $1 / 2 \leq \theta \leq 1$. There exists a suitable positive constant $C$ such that

$$
\limsup _{T \rightarrow+\infty} \frac{1}{T} \int_{0}^{T}\left\|\nabla A_{\theta}^{1 / 2} D_{N, \theta}^{1 / 2} \boldsymbol{w}\right\|^{2}\left\|A_{\theta}^{1 / 2} D_{N, \theta}^{1 / 2} \boldsymbol{w}\right\|^{2 \mu} d t \leq C \frac{K_{1}^{1+\mu}}{\nu^{1+\mu} \lambda_{1}^{\mu}},
$$

where $K_{1}$ is defined in (22).

Proof. From estimate (24) we find in particular

from which

$$
\left\|A_{\theta}^{1 / 2} D_{N, \theta}^{1 / 2} \boldsymbol{w}(t)\right\|^{2} \leq k_{0} \mathrm{e}^{-\nu \lambda_{1} t}+\frac{K_{1}}{\nu \lambda_{1}}
$$

$$
\left\|A_{\theta}^{1 / 2} D_{N, \theta}^{1 / 2} \boldsymbol{w}(t)\right\|^{2 \mu} \leq C\left\{k_{0}^{\mu} \mathrm{e}^{-\nu \mu \lambda_{1} t}+\left(\frac{K_{1}}{\nu \lambda_{1}}\right)^{\mu}\right\} .
$$

We note that the constant $C$ in the inequality above can be chosen to be independent of $\mu$, since $0 \leq \mu \leq 2$ as $1 / 2 \leq \theta \leq 1$, see Remark 6.4. Hence

$$
\begin{aligned}
\limsup _{T \rightarrow+\infty} \frac{1}{T} \int_{0}^{T}\left\|\nabla A_{\theta}^{1 / 2} D_{N, \theta}^{1 / 2} \boldsymbol{w}\right\|^{2}\left\|A_{\theta}^{1 / 2} D_{N, \theta}^{1 / 2} \boldsymbol{w}\right\|^{2 \mu} d t \\
\leq C k_{0}^{\mu} \limsup _{T \rightarrow+\infty} \frac{1}{T} \int_{0}^{T} e^{-\nu \mu \lambda_{1} t}\left\|\nabla A_{\theta}^{1 / 2} D_{N, \theta}^{1 / 2} \boldsymbol{w}\right\|^{2} d t \\
\quad+C\left(\frac{K_{1}}{\nu \lambda_{1}}\right)^{\mu} \limsup _{T \rightarrow+\infty} \frac{1}{T} \int_{0}^{T}\left\|\nabla A_{\theta}^{1 / 2} D_{N, \theta}^{1 / 2} \boldsymbol{w}\right\|^{2} d t=I_{1}+I_{2} .
\end{aligned}
$$

To estimate $I_{1}$ we use (26) (with the constant $C$ in (26) equal to $\frac{1}{\mu}$ ) to find

$$
\int_{0}^{T} e^{-\nu \lambda_{1} \mu t}\left\|\nabla A_{\theta}^{1 / 2} D_{N, \theta}^{1 / 2} \boldsymbol{w}(s)\right\|^{2} d s \leq \frac{K_{1}}{\mu \nu^{2} \lambda_{1}}+\frac{k_{0}}{\nu},
$$

$k_{0}, K_{1}$ being defined in (21), (22), hence

$$
I_{1}=C k_{0}^{\mu} \limsup _{T \rightarrow+\infty} \frac{1}{T} \int_{0}^{T} e^{-\nu \mu \lambda_{1} t}\left\|\nabla A_{\theta}^{1 / 2} D_{N, \theta}^{1 / 2} \boldsymbol{w}(t)\right\|^{2} d t=0 .
$$

To estimate $I_{2}$, we use (25) with $t=0, r=T$ to find

$$
\int_{0}^{T}\left\|\nabla A_{\theta}^{1 / 2} D_{N, \theta}^{1 / 2} \boldsymbol{w}(t)\right\|^{2} d t \leq \frac{T K_{1}+k_{1}}{\nu},
$$


where $k_{1}$ is defined in (23), from which

$$
\begin{aligned}
I_{2} & =C\left(\frac{K_{1}}{\nu \lambda_{1}}\right)^{\mu} \limsup _{T \rightarrow+\infty} \frac{1}{T} \int_{0}^{T}\left\|\nabla A_{\theta}^{1 / 2} D_{N, \theta}^{1 / 2} \boldsymbol{w}\right\|^{2} d t \\
& \leq C\left(\frac{K_{1}}{\nu \lambda_{1}}\right)^{\mu} \limsup _{T \rightarrow+\infty} \frac{1}{T} \frac{T K_{1}+k_{1}}{\nu}=C \frac{K_{1}^{1+\mu}}{\nu^{1+\mu} \lambda_{1}^{\mu}} .
\end{aligned}
$$

Then (80) follows from (81), (82) and (83).

\subsection{Estimate by the Grashoff number (proof of Theorem 1.2)}

Using (78), let us firstly derive an estimate of $M$ in terms of the Grashoff number $G$ defined in (6). Comparing $G$ with $K_{1}$ defined in (22), through the Poincaré inequality (where we use that $\lambda_{1}=L^{-2}$ ), we get

$$
\begin{aligned}
K_{1} & =\frac{\left\|\Lambda^{-1} A_{\theta}^{-1 / 2} D_{N, \theta}^{1 / 2} \boldsymbol{f}\right\|^{2}}{\nu} \leq \frac{\lambda_{1}^{-1}\left\|A_{\theta}^{-1 / 2} D_{N, \theta}^{1 / 2} \boldsymbol{f}\right\|^{2}}{\nu}=\frac{L^{2}\left\|A_{\theta}^{-1 / 2} D_{N, \theta}^{1 / 2} \boldsymbol{f}\right\|^{2}}{\nu} \\
& =\frac{\nu^{3} G^{2}}{L}
\end{aligned}
$$

Using (84) in (78) then gives

$$
\begin{aligned}
X_{M} & \geq \frac{\nu \lambda_{1} M^{5 / 3}}{C}-M^{(1-\sigma) s} C_{1} C \frac{\nu^{2+2 \mu} G^{2+2 \mu}}{L^{1+\mu} \lambda_{1}^{\mu}} \\
& =\frac{\nu L^{-2} M^{5 / 3}}{C}-M^{(1-\sigma) s} C_{1} C \nu^{2+2 \mu} G^{2+2 \mu} L^{\mu-1},
\end{aligned}
$$

from which $X_{M}>0$ holds true provided that

$$
M>C\left(C_{1} \nu^{1+2 \mu} L^{1+\mu}\right)^{\frac{3}{5+3(\sigma-1) s}} G^{\frac{6+6 \mu}{5+3(\sigma-1) s}} .
$$

\subsection{Estimate by mean dissipation length (proof of Theorem 1.3)}

In order to get the desired estimate, we are going to produce an estimate from below of $X_{M}$, defined in (68), different from the one provided by Lemma 6.5.

We will prove the following result.

Lemma 6.8. Assume that $1 / 2<\theta \leq 1$. Then the following estimate

$$
X_{M} \geq \frac{\nu M^{5 / 3}}{C L^{2}}-\frac{C(N+1)^{2 \gamma} M^{2(1-\gamma)} L^{3+6 / q} \nu}{\alpha^{4 \gamma \theta} \ell_{d}^{4}}
$$

holds true, where it is set

$$
\gamma=\frac{1}{3-2 \theta} \quad \text { and } \quad \frac{1}{2}+\frac{1}{q}=\gamma .
$$

Proof. In view of (67) we firstly get an estimate of $\mathcal{R}_{M}(t)$, by arguing exactly as we did to obtain the estimate (74) in the proof of Lemma 6.3, but making a different choice of the exponents; namely, by the Hölder inequality and (17) we get for each $1 / 2<\theta \leq 1$ 


$$
\begin{aligned}
\left|\mathcal{R}_{M}(t)\right| \leq & \int_{\mathbb{T}^{3}}\left(\sum_{i=1}^{M}\left|D_{N, \theta} \boldsymbol{\varphi}_{i}(t)\right|^{2}\right)^{\gamma}\left(\sum_{i=1}^{M}\left|D_{N, \theta} \boldsymbol{\varphi}_{i}(t)\right|^{2}\right)^{1-\gamma}\left|\nabla D_{N, \theta} \boldsymbol{w}\right| \mathrm{d} \boldsymbol{x} \\
\leq & \left\|\left(\sum_{i=1}^{M}\left|D_{N, \theta} \boldsymbol{\varphi}_{i}(t)\right|^{2}\right)^{\gamma}\right\|\left\|_{L^{\infty}}\right\|\left(\sum_{i=1}^{M}\left|D_{N, \theta} \boldsymbol{\varphi}_{i}(t)\right|^{2}\right)^{\frac{1}{1-\gamma}} \|_{L^{1-\gamma}} \\
& \times\left\|\nabla D_{N, \theta} \boldsymbol{w}\right\|\left|\mathbb{T}^{3}\right|^{1 / q} \\
\leq & C\left\|\sum_{i=1}^{M}\left|D_{N, \theta} \boldsymbol{\varphi}_{i}(t)\right|^{2}\right\|_{L^{\infty}}^{\gamma}\left(\sum_{i=1}^{M}\left\|D_{N, \theta} \boldsymbol{\varphi}_{i}(t)\right\|^{2}\right)^{1-\gamma}\left\|\nabla D_{N, \theta} \boldsymbol{w}\right\| L^{3 / q} \\
\leq & C\left\|\sum_{i=1}^{M}\left|D_{N, \theta} \boldsymbol{\varphi}_{i}(t)\right|^{2}\right\|_{L^{\infty}}^{\gamma}\left(\sum_{i=1}^{M}\left\|A_{\theta}^{1 / 2} D_{N, \theta}^{1 / 2} \boldsymbol{\varphi}_{i}(t)\right\|^{2}\right)^{1-\gamma} \\
& \times\left\|\nabla D_{N, \theta} \boldsymbol{w}\right\| L^{3 / q} \\
= & C\left\|\sum_{i=1}^{M}\left|D_{N, \theta} \boldsymbol{\varphi}_{i}(t)\right|^{2}\right\|_{L^{\infty}}^{\gamma} M^{1-\gamma}\left\|\nabla D_{N, \theta} \boldsymbol{w}\right\| L^{3 / q},
\end{aligned}
$$

where the exponents $\gamma$ and $q$ are defined by means of (86). Notice that the second relation in (86) is just the needed constraint in order that the Hölder inequality applies with the exponents $\gamma$ and $q$ above. Notice also that $\gamma=\frac{1}{3-2 \theta}$ is an increasing function of $\theta$, hence

$$
1 / 2<\gamma \leq 1, \quad \text { as long as } 1 / 2<\theta \leq 1
$$

Since in particular $\gamma=1$, as long as $\theta=1$, the same observation about the estimate (75) applies to the estimate above when $\theta=1$. In this case the splitting of $\sum_{i=1}^{M}\left|D_{N, \theta} \boldsymbol{\varphi}_{i}(t)\right|^{2}$ into the $\gamma$ and $(1-\gamma)$-powers becomes trivial: the $L^{\infty}$ and $L^{\frac{1}{1-\gamma}}$-norms of those two powers reduce to the $L^{\infty}$-norm of the whole $\sum_{i=1}^{M}\left|D_{N, \theta} \varphi_{i}(t)\right|^{2}$ (hence $M^{1-\gamma}=1$ ) and $q=2$. An explicit calculation gives that

$$
q=\frac{2(3-2 \theta)}{2 \theta-1}
$$

for arbitrary $1 / 2<\theta \leq 1$.

Using (17) and (75) to estimate respectively the $L^{2}$-norm of $\nabla D_{N, \theta} \boldsymbol{w}$ and the $L^{\infty}$-norm of $\sum_{i=1}^{M}\left|D_{N, \theta} \boldsymbol{\varphi}_{i}(t)\right|^{2}$ in the right-hand side of (87), we obtain

$$
\left|\mathcal{R}_{M}(t)\right| \leq \frac{C(N+1)^{\gamma}}{\alpha^{2 \gamma \theta} \nu^{\gamma(3 / 2-\theta)}}\left(Q_{M}(t)\right)^{\gamma(3 / 2-\theta)} M^{1-\gamma}\left\|\nabla A_{\theta}^{1 / 2} D_{N, \theta}^{1 / 2} \boldsymbol{w}\right\| L^{3 / q} .
$$

Again, as done in the proof of Lemma 6.3 we apply to the last expression above the Young inequality $a b \leq \frac{a^{r}}{r}+\frac{b^{s}}{s}$, where the conjugate exponents $r=\frac{1}{\gamma(3 / 2-\theta)}=\frac{2}{\gamma(3-2 \theta)}$ and $s=\frac{1}{1-\gamma(3 / 2-\theta)}=\frac{2}{2-3 \gamma+2 \gamma \theta}$ are chosen in such a way that $\frac{a^{r}}{r}=\frac{Q_{M}(t)}{2}$; then we get 


$$
\left|R_{M}(t)\right| \leq \frac{Q_{M}(t)}{2}+C \frac{(N+1)^{s \gamma} L^{3 s / q} M^{s(1-\gamma)}}{\alpha^{2 s \gamma \theta} \nu^{s \gamma(3 / 2-\theta)}}\left\|\nabla A_{\theta}^{1 / 2} D_{N, \theta}^{1 / 2} \boldsymbol{w}\right\|^{s} .
$$

Now the crucial point is to observe that, with the choice of $\gamma$ made in (86), we get $\gamma(3-2 \theta)=1$, thus $s=r=2$. Hence, the estimate (89) becomes

$$
\left|R_{M}(t)\right| \leq \frac{Q_{M}(t)}{2}+C \frac{(N+1)^{2 \gamma} L^{6 / q} M^{2(1-\gamma)}}{\alpha^{4 \gamma \theta} \nu}\left\|\nabla A_{\theta}^{1 / 2} D_{N, \theta}^{1 / 2} \boldsymbol{w}\right\|^{2} .
$$

For the rest, the proof follows the same lines of Lemma 6.5. Firstly, from (67) we find

$$
\begin{aligned}
\mathcal{T}_{M}(t) & =Q_{M}(t)+\mathcal{R}_{M}(t) \geq Q_{M}(t)-\left|\mathcal{R}_{M}(t)\right| \\
& \geq \frac{Q_{M}(t)}{2}-C \frac{(N+1)^{2 \gamma} L^{6 / q} M^{2(1-\gamma)}}{\alpha^{4 \gamma \theta} \nu}\left\|\nabla A_{\theta}^{1 / 2} D_{N, \theta}^{1 / 2} \boldsymbol{w}\right\|^{2} .
\end{aligned}
$$

Then we calculate

$$
\begin{aligned}
X_{M}= & \liminf _{T \rightarrow+\infty} \frac{1}{T} \int_{0}^{T} \mathcal{T}_{M}(t) d t \\
\geq & \liminf _{T \rightarrow+\infty} \frac{1}{T} \int_{0}^{T} \frac{Q_{M}(t)}{2} d t \\
& -C \frac{(N+1)^{2 \gamma} L^{6 / q} M^{2(1-\gamma)}}{\alpha^{4 \gamma \theta} \nu} \limsup _{T \rightarrow+\infty} \frac{1}{T} \int_{0}^{T}\left\|\nabla A_{\theta}^{1 / 2} D_{N, \theta}^{1 / 2} \boldsymbol{w}\right\|^{2} d t \\
= & \liminf _{T \rightarrow+\infty} \frac{1}{T} \int_{0}^{T} \frac{Q_{M}(t)}{2} d t \\
& -C \frac{(N+1)^{2 \gamma} L^{6 / q} M^{2(1-\gamma)}}{\alpha^{4 \gamma \theta} \nu^{2}} \limsup _{T \rightarrow+\infty} \frac{1}{T} \int_{0}^{T} \nu\left\|\nabla A_{\theta}^{1 / 2} D_{N, \theta}^{1 / 2} \boldsymbol{w}\right\|^{2} d t .
\end{aligned}
$$

We use (69) to estimate from below $\liminf _{T \rightarrow+\infty} \frac{1}{T} \int_{0}^{T} \frac{Q_{M}(t)}{2} d t$ (recall that $\lambda_{1}=$ $\left.L^{-2}\right)$ by

$$
\liminf _{T \rightarrow+\infty} \frac{1}{T} \int_{0}^{T} \frac{Q_{M}(t)}{2} d t \geq \frac{\nu M^{5 / 3}}{C L^{2}}
$$

and (9) to estimate $\limsup _{T \rightarrow+\infty} \frac{1}{T} \int_{0}^{T} \nu\left\|\nabla A_{\theta}^{1 / 2} D_{N, \theta}^{1 / 2} \boldsymbol{w}\right\|^{2} d t$ by

$$
\limsup _{T \rightarrow+\infty} \frac{1}{T} \int_{0}^{T} \nu\left\|\nabla A_{\theta}^{1 / 2} D_{N, \theta}^{1 / 2} \boldsymbol{w}(t)\right\|^{2} d t \leq \frac{L^{3} \nu^{3}}{\ell_{d}^{4}} .
$$

We use the last two inequalities above to bound the right-hand side of (90) and to obtain

$$
\begin{aligned}
X_{M} \geq & \frac{\nu M^{5 / 3}}{C L^{2}}-C \frac{(N+1)^{2 \gamma} L^{6 / q} M^{2(1-\gamma)}}{\alpha^{4 \gamma \theta} \nu^{2}} \frac{L^{3} \nu^{3}}{\ell_{d}^{4}}=\frac{\nu M^{5 / 3}}{C L^{2}} \\
& -C \frac{(N+1)^{2 \gamma} L^{3+6 / q} M^{2(1-\gamma)} \nu}{\alpha^{4 \gamma \theta} \ell_{d}^{4}},
\end{aligned}
$$

that is just the estimate (85). 
Let us observe that in view of $(88)$, the exponent $2(1-\gamma)$ in the power of $M$ appearing in the second term of the right-hand side of (85) is smaller than 1 . Then, in view (85), we see that $X_{M}>0$ holds true provided that

$$
M^{5 / 3-2(1-\gamma)}>\frac{C(N+1)^{2 \gamma}}{\alpha^{4 \gamma \theta}} \frac{L^{5+6 / q}}{\ell_{d}^{4}}=\frac{C(N+1)^{2 \gamma}}{\alpha^{4 \gamma \theta}}\left(\frac{L}{\ell_{d}}\right)^{4} L^{1+6 / q},
$$

which gives

$$
\begin{aligned}
M & >C\left[\frac{(N+1)^{2 \gamma}}{\alpha^{4 \gamma \theta}} L^{1+6 / q}\right]^{\frac{3}{5-6(1-\gamma)}}\left(\frac{L}{\ell_{d}}\right)^{\frac{12}{5-6(1-\gamma)}} \\
& =C\left[(N+1)^{\gamma}\left(\frac{L}{\alpha}\right)^{2 \gamma \theta}\left(\frac{L}{\ell_{d}}\right)^{2}\right]^{\frac{6}{6 \gamma-1}}
\end{aligned}
$$

where we have used the identity $1+\frac{6}{q}=4 \gamma \theta$.

\section{Appendix: Some useful formulas and inequalities}

Lemma A.1. Let $\boldsymbol{a}, \boldsymbol{b}, \boldsymbol{c}$ be sufficiently smooth vector fields such that $\nabla \cdot \boldsymbol{a}=0$. Then,

$$
\begin{aligned}
& \nabla \cdot(\boldsymbol{b} \otimes \boldsymbol{a})=(\boldsymbol{a} \cdot \nabla) \boldsymbol{b} \\
& ((\boldsymbol{a} \cdot \nabla) \boldsymbol{b}, \boldsymbol{c})=-((\boldsymbol{a} \cdot \nabla) \boldsymbol{c}, \boldsymbol{b}) \\
& (\nabla \cdot(\boldsymbol{b} \otimes \boldsymbol{a}), \boldsymbol{b})=0 .
\end{aligned}
$$

The following estimates are particular cases of the well known GagliardoNirenberg inequality (see [16]), where we make use of the equivalence of norms given in Remark 2.4 .

Lemma A.2. Let us assume that $1 / 2 \leq \theta \leq 1$ and define $\sigma(\theta)$ by setting

$$
\sigma(\theta):= \begin{cases}\frac{1+\theta}{3-2 \theta}, & \text { if } 1 / 2 \leq \theta<2 / 3 \\ 1, & \text { if } 2 / 3 \leq \theta \leq 1\end{cases}
$$

There exists a positive constant $C$ such that for every $\boldsymbol{u}=\boldsymbol{u}(\boldsymbol{x})$ sufficiently smooth vector or matrix-valued function we have

$$
\begin{aligned}
\|\boldsymbol{u}\|_{\mathrm{L}^{3}} & \leq C\left\|A_{\theta}^{1 / 2} \boldsymbol{u}\right\|^{a}\|\boldsymbol{u}\|^{1-a}, \\
\|\nabla \boldsymbol{u}\|_{\mathrm{L}^{3}} & \leq C\left\|\nabla A_{\theta} \boldsymbol{u}\right\|^{b}\left\|A_{\theta}^{1 / 2} \boldsymbol{u}\right\|^{1-b}, \\
\left\|A_{\theta} \boldsymbol{u}\right\|_{\mathrm{L}^{3}} & \leq C\left\|\nabla A_{\theta} u\right\|^{c}\left\|A_{\theta}^{1 / 2} \boldsymbol{u}\right\|^{1-c}, \\
\|\nabla \boldsymbol{u}\|_{\mathrm{L}^{p}} & \leq C\left\|\nabla A_{\theta}^{1 / 2} \boldsymbol{u}\right\|^{\beta}\left\|A_{\theta}^{1 / 2} \boldsymbol{u}\right\|^{1-\beta},
\end{aligned}
$$

where

$$
\begin{array}{ll}
a=\frac{1}{2 \theta}, \quad b=\frac{3}{1+\theta}\left(\frac{1}{2}-\frac{\theta}{3}\right), & c=\frac{\theta+1 / 2}{\theta+1} \\
p=\frac{6}{1+6 \sigma(\theta)-2(1+2 \sigma(\theta)) \theta}, & \beta=2-3 \sigma(\theta)+2 \sigma(\theta) \theta .
\end{array}
$$


Note that the exponents $a, b, c$ involved in the estimates (95)-(97) and computed above satisfy the constraints required by the Gagliardo-Nirenberg inequality, in particular

$$
1 / 2 \leq a \leq 1, \quad \frac{1-\theta}{1+\theta} \leq b \leq 1, \quad \frac{\theta}{1+\theta} \leq c \leq 1
$$

for every $1 / 2 \leq \theta \leq 1$. Moreover, we have

$$
1 / 4 \leq b \leq 2 / 3, \quad 2 / 3 \leq c \leq 3 / 4 .
$$

As regards to the last inequality (98), due to the formula (94), it is worth considering separately the two cases below:

1 st case: for $1 / 2 \leq \theta<2 / 3$ the expressions in (100) become

$$
p=2, \quad \beta=1-\theta \text {. }
$$

2nd case: for $2 / 3 \leq \theta \leq 1$ the expressions in (100) become

$$
p=\frac{6}{7-6 \theta}, \quad \beta=2 \theta-1 .
$$

Notice that $p$ defined in (103) is an increasing function of $\theta$ and we have

$$
2 \leq p \leq 6, \quad \text { as } 2 / 3 \leq \theta \leq 1 .
$$

Notice also that in both the previous cases the exponents $p$ and $\beta$ in (102)(103) satisfy the constraints required by the Gagliardo-Nirenberg inequality, in particular $1-\theta \leq \beta \leq 1$ for every $1 / 2 \leq \theta \leq 1$.

The fractional version of Agmon's inequality can be found in [10] (p. 37, Lemma 4.9; the proof is for $\mathbb{R}^{n}$, but can be easily adapted to the periodic case by resorting to the Fourier series instead of the Fourier transform).

Lemma A.3. (Fractional Agmon's inequality) Let us assume that

$$
0<s_{1}<n / 2<s_{2},
$$

where $n$ is the space dimension of the periodic box domain $D$, and let $t \in(0,1)$ such that $n / 2=(1-t) s_{1}+t s_{2}$. Then there exists a constant $C=C\left(n, s_{1}, s_{2}\right)$ such that

$$
\|\boldsymbol{u}\|_{\mathrm{L}^{\infty}} \leq C\|\boldsymbol{u}\|_{\mathrm{H}^{s_{1}}}^{1-t}\|\boldsymbol{u}\|_{\mathrm{H}^{s_{2}}}^{t}
$$

where the norms refer to the space $D$.

In particular, if $D=\mathbb{T}^{3}$ and $1 / 2<\theta \leq 1$, we have

$$
\|\boldsymbol{u}\|_{\mathrm{L}^{\infty}} \leq \frac{C}{\alpha^{\theta}}\left\|A_{\theta}^{1 / 2} \boldsymbol{u}\right\|^{\theta-1 / 2}\left\|\nabla A_{\theta}^{1 / 2} \boldsymbol{u}\right\|^{3 / 2-\theta} .
$$

The final estimate can be easily obtained observing that

$$
\begin{aligned}
\|\boldsymbol{u}\|_{\theta, 2} & \leq \frac{1}{\alpha^{\theta}}\left\|A_{\theta}^{1 / 2} \boldsymbol{u}\right\|, \\
\|\boldsymbol{u}\|_{\theta+1,2} & \leq \frac{1}{\alpha^{\theta}}\left\|\nabla A_{\theta}^{1 / 2} \boldsymbol{u}\right\|,
\end{aligned}
$$

as it is easily seen using Fourier expansions.

The following inequality generalizes Lemma 5, Estimate (28), in FoiasHolm-Titi [11] and is used in Sect. 6. 
Lemma A.4. Assume that $1 / 2<\theta \leq 1$. Let $\boldsymbol{\varphi}_{j}, j=1, \ldots, M$, be orthonormal vector functions with respect to the scalar product $[\cdot, \cdot \cdot]$ defined in (61), and set

$$
Q_{M}:=\nu \sum_{j=1}^{M}\left\|\nabla A_{\theta}^{1 / 2} D_{N, \theta}^{1 / 2} \boldsymbol{\varphi}_{j}\right\|^{2} .
$$

Then there exists $C>0$ (independent, in particular, of $M$ ) such that

$$
\sum_{j=1}^{M}\left|D_{N, \theta} \boldsymbol{\varphi}_{j}(\boldsymbol{x})\right|^{2} \leq \frac{C}{\alpha^{2 \theta}}(N+1)\left(\frac{Q_{M}}{\nu}\right)^{3 / 2-\theta}
$$

for each $\boldsymbol{x} \in \mathbb{T}^{3}$.

Proof. For fixed real numbers $\xi_{1}, \ldots, \xi_{M}$ (that we will choose later on), by using (104) and then (18)-(19) with $\boldsymbol{w}=\sum_{j=1}^{M} \xi_{j} \boldsymbol{\varphi}_{j}$, we have

$$
\begin{aligned}
\left|\sum_{j=1}^{M} \xi_{j} D_{N, \theta} \boldsymbol{\varphi}_{j}(\boldsymbol{x})\right|^{2} & \\
\leq & \frac{C}{\alpha^{2 \theta}}\left\|\sum_{j=1}^{M} \xi_{j} A_{\theta}^{1 / 2} D_{N, \theta} \boldsymbol{\varphi}_{j}\right\|^{2 \theta-1}\left\|\sum_{j=1}^{M} \xi_{j} \nabla A_{\theta}^{1 / 2} D_{N, \theta} \boldsymbol{\varphi}_{j}\right\|^{3-2 \theta} \\
\leq & \frac{C(N+1)}{\alpha^{2 \theta}}\left\|\sum_{j=1}^{M} \xi_{j} A_{\theta}^{1 / 2} D_{N, \theta}^{1 / 2} \boldsymbol{\varphi}_{j}\right\|^{2 \theta-1}\left\|\sum_{j=1}^{M} \xi_{j} \nabla A_{\theta}^{1 / 2} D_{N, \theta}^{1 / 2} \boldsymbol{\varphi}_{j}\right\|^{3-2 \theta} \\
\leq & \frac{C(N+1)}{\alpha^{2 \theta}}\left(\sum_{i=1}^{M} \xi_{i} A_{\theta}^{1 / 2} D_{N, \theta}^{1 / 2} \boldsymbol{\varphi}_{i}, \sum_{j=1}^{M} \xi_{j} A_{\theta}^{1 / 2} D_{N, \theta}^{1 / 2} \boldsymbol{\varphi}_{j}\right)^{\theta-\frac{1}{2}} \\
& \times\left(\sum_{j=1}^{M}\left|\xi_{j}\right|\left\|\nabla A_{\theta}^{1 / 2} D_{N, \theta}^{1 / 2} \boldsymbol{\varphi}_{j}\right\|\right)^{3-2 \theta} \\
\leq & \frac{C(N+1)}{\alpha^{2 \theta}}\left[\sum_{i, j=1}^{M} \xi_{i} \xi_{j}\left(A_{\theta}^{1 / 2} D_{N, \theta}^{1 / 2} \boldsymbol{\varphi}_{i}, A_{\theta}^{1 / 2} D_{N, \theta}^{1 / 2} \boldsymbol{\varphi}_{j}\right)\right]^{\theta-1 / 2}\left(\sum_{j=1}^{M} \xi_{j}^{2}\right)^{3 / 2-\theta} \\
& \times\left(\sum_{j=1}^{M}\left\|\nabla A_{\theta}^{1 / 2} D_{N, \theta}^{1 / 2} \boldsymbol{\varphi}_{j}\right\|^{2}\right)^{3 / 2-\theta}
\end{aligned}
$$

where we have used the discrete Cauchy-Schwarz inequality. If we denote by $\delta_{i j}$ the Kronecker symbol, using the orthonormality of the functions $\boldsymbol{\varphi}_{j}$ and the definition of $Q_{M}$ above, we obtain 


$$
\begin{aligned}
\left|\sum_{j=1}^{M} \xi_{j} D_{N, \theta} \varphi_{j}(\boldsymbol{x})\right|^{2} \leq & \frac{C(N+1)}{\alpha^{2 \theta}}\left[\sum_{i, j=1}^{M} \xi_{i} \xi_{j} \delta_{i j}\right]^{\theta-1 / 2}\left(\sum_{j=1}^{M} \xi_{j}^{2}\right)^{3 / 2-\theta} \\
& \times\left(\frac{Q_{M}}{\nu}\right)^{3 / 2-\theta} \\
\leq & \frac{C(N+1)}{\alpha^{2 \theta}}\left(\sum_{j=1}^{M} \xi_{j}^{2}\right)\left(\frac{Q_{M}}{\nu}\right)^{3 / 2-\theta}
\end{aligned}
$$

If we take $\xi_{j}$ equal to the $i$-th component of $D_{N, \theta} \boldsymbol{\varphi}_{j}$, for a fixed $i \in$ $\{1,2,3\}$, i.e. $\xi_{j}=D_{N, \theta} \varphi_{i, j}(\boldsymbol{x})$, and sum over $i=1,2,3$, we deduce

$$
\begin{aligned}
\sum_{i=1}^{3}\left|\sum_{j=1}^{M} D_{N, \theta} \varphi_{i, j}(\boldsymbol{x}) D_{N, \theta} \boldsymbol{\varphi}_{j}(\boldsymbol{x})\right|^{2} \leq & \frac{C}{\alpha^{2 \theta}}(N+1)\left(\sum_{i=1}^{3} \sum_{j=1}^{M}\left(D_{N, \theta} \varphi_{i, j}(\boldsymbol{x})\right)^{2}\right) \\
& \times\left(\frac{Q_{M}}{\nu}\right)^{3 / 2-\theta} .
\end{aligned}
$$

On the other hand, we notice that the left-hand side is greater than or equal to

$$
\sum_{i=1}^{3}\left(\sum_{j=1}^{M}\left(D_{N, \theta} \varphi_{i, j}(\boldsymbol{x})\right)^{2}\right)^{2} \geq \frac{1}{C}\left(\sum_{i=1}^{3} \sum_{j=1}^{M}\left(D_{N, \theta} \varphi_{i, j}(\boldsymbol{x})\right)^{2}\right)^{2}
$$

where $C>1$ depends only on the space dimension. Combining this result with the previous inequality, we obtain

$$
\begin{aligned}
\left(\sum_{i=1}^{3} \sum_{j=1}^{M}\left(D_{N, \theta} \varphi_{i, j}(\boldsymbol{x})\right)^{2}\right)^{2} \leq & \frac{C}{\alpha^{2 \theta}}(N+1)\left(\sum_{i=1}^{3} \sum_{j=1}^{M}\left(D_{N, \theta} \varphi_{i, j}(\boldsymbol{x})\right)^{2}\right) \\
& \times\left(\frac{Q_{M}}{\nu}\right)^{3 / 2-\theta} .
\end{aligned}
$$

The conclusion follows by simplifying. $[14])$.

Another useful tool is represented by the Lieb-Thirring inequality (see

Lemma A.5. (Lieb-Thirring inequality) Let us assume that $\boldsymbol{\theta}_{j}$, with $j=1, \ldots$, $M$, are orthonormal functions in $\mathrm{L}_{\sigma}^{2}\left(\mathbb{T}^{3}\right)$. Then there exists $C>0$ (independent of $M$ ) such that

$$
\int_{\mathbb{T}^{3}}\left(\sum_{j=1}^{M} \boldsymbol{\theta}_{j}(\boldsymbol{x}) \cdot \boldsymbol{\theta}_{j}(\boldsymbol{x})\right)^{5 / 3} \mathrm{~d} \boldsymbol{x} \leq C \sum_{j=1}^{M} \int_{\mathbb{T}^{3}} \nabla \boldsymbol{\theta}_{j}(\boldsymbol{x}): \nabla \boldsymbol{\theta}_{j}(\boldsymbol{x}) \mathrm{d} \boldsymbol{x},
$$

where for matrices $A=\left(a_{i, j}\right), B=\left(b_{i, j}\right)$ it is set $A: B:=\sum_{i=1}^{M} \sum_{j=1}^{M} a_{i, j} b_{i, j}$. 


\section{References}

[1] Ali, H.: Global well-posedness for deconvolution magnetohydrodynamics models with fractional regularization. Methods Appl. Anal. 20(3), 211-236 (2013)

[2] Berselli, L.C., Iliescu, T., Layton, W.J.: Mathematics of Large Eddy Simulation of Turbulent Flows. Sci. Comput. Springer, Berlin (2006)

[3] Berselli, L.C., Catania, D., Lewandowski, R.: Convergence of approximate deconvolution models to the mean magnetohydrodynamics equations: analysis of two models. J. Math. Anal. Appl. 401, 864-880 (2013)

[4] Berselli, L.C., Lewandowski, R.: Convergence of approximate deconvolution models to the mean Navier-Stokes equations. Ann. Inst. H. Poincaré Anal. Non Linéaire 29(2), 171-198 (2012)

[5] Cao, Y., Lunasin, E.M., Titi, E.S.: Global well-posedness of the threedimensional viscous and inviscid simplified Bardina turbulence models. Commun. Math. Sci. 4(4), 823-848 (2006)

[6] Catania, D.: Global attractor and determining modes for a hyperbolic MHD turbulence model. J. Turbul. 12(40), 1-20 (2011)

[7] Catania, D.: Finite dimensional global attractor for 3D MHD- $\alpha$ models: a comparison. J. Math. Fluid Mech. 14, 95-115 (2012)

[8] Catania, D., Secchi, P.: Global existence and finite dimensional global attractor for a 3D double viscous MHD- $\alpha$ model. Commun. Math. Sci. 8(4), 1021$1040(2010)$

[9] Catania, D., Secchi, P.: Global existence for two regularized MHD models in three space-dimension. Portugal. Math. (N.S.) 68(1), 41-52 (2011)

[10] Constantin, P., Foias, C.: Navier-Stokes Equations. Chicago Lectures in Mathematics. University of Chicago Press (1988)

[11] Foias, C., Holm, D.D., Titi, E.S.: The three dimensional viscous Camassa-Holm equations, and their relation to the Navier-Stokes equations and turbulence theory. J. Dyn. Differ. Equ. 14(1), 1-35 (2002)

[12] Galdi, G.P.: An Introduction to the Navier-Stokes Initial-Boundary Value Problem. Fundamental Directions in Fluid Mechanics. Advances in Mathematical Fluid Mechanics, pp. 1-70. Birkhäuser, Basel (2000)

[13] Layton, W., Lewandowski, R.: On a well-posed turbulence model. Discret. Contin. Dyn. Syst. B 6, 111-128 (2006)

[14] Lieb, E., Thirring, W.: Inequalities for the Moments of the Eigenvalues of the Schrödinger Hamiltonian and Their Relation to Sobolev Inequalities. In: Lieb, E., Simon, B., Wightman, A.S. (eds.) Studies in Mathematical Physics: Essays in Honor of V. Bargman, pp. 226-303. Princeton University Press, Princeton (1976) 
[15] Mohseni, K., Kosović, B., Shkoller, S., Marsden, J.: Numerical simulations of the Lagrangian averaged Navier-Stokes-alpha-like models. Phys. Fluids 15(2), 524544 (2003)

[16] Nirenberg, L.: On elliptic partial differential equations. Ann. Sc. Norm. Sup. Pisa 13(3), 115-162 (1959)

[17] Sell, G.R., You, Y.: Dynamics of Evolutionary Equations. Applied Mathematical Sciences, vol. 143. Springer, Berlin (2002)

[18] Stolz, S., Adams, N.A.: An approximate deconvolution procedure for large-eddy simulation. Phys. Fluids 11(7), 1699-1701 (1999)

[19] Temam, R.: Infinite-Dimensional Dynamical Systems in Mechanics and Physics, 2nd edn. Applied Mathematical Sciences, vol. 68. Springer, New York (1997)

Davide Catania, Alessandro Morando and Paola Trebeschi

DICATAM

Sezione Matematica

Università degli Studi di Brescia

Via Valotti 9

25133 Brescia

Italy

e-mail: davide.catania@unibs.it

Alessandro Morando

e-mail: alessandro.morando@unibs.it

Paola Trebeschi

e-mail: paola.trebeschi@unibs.it

Davide Catania

Facoltà di Ingegneria

Università degli Studi eCampus

Via Isimbardi 10

22060 Novedrate, CO

Italy

Received: 5 September 2014.

Accepted: 9 December 2014. 